Commun. Korean Math. Soc. 26 (2011), No. 3, pp. 349-371

DOI 10.4134/CKMS.2011.26.3.349

\title{
FUZZY SUBGROUPS BASED ON FUZZY POINTS
}

\author{
Young Bae Jun, Min Su Kang, and Chul Hwan Park
}

\begin{abstract}
Using the "belongs to" relation and "quasi-coincident with" relation between a fuzzy point and a fuzzy subgroup, Bhakat and Das, in 1992 and 1996, initiated general types of fuzzy subgroups which are a generalization of Rosenfeld's fuzzy subgroups. In this paper, more general notions of "belongs to" and "quasi-coincident with" relation between a fuzzy point and a fuzzy set are provided, and more general formulations of general types of fuzzy (normal) subgroups by Bhakat and Das are discussed. Furthermore, general type of coset is introduced, and related fundamental properties are investigated.
\end{abstract}

\section{Introduction}

To solve complicated problems in economics, engineering, and environment, we can not successfully use classical methods because of various uncertainties typical for those problems. There are three theories: theory of probability, theory of fuzzy sets, and the interval mathematics which we can consider as mathematical tools for dealing with uncertainties. But all these theories have their own difficulties. Uncertainties can not be handled using traditional mathematical tools but may be dealt with using a wide range of existing theories such as probability theory, theory of (intuitionistic) fuzzy sets, theory of vague sets, theory of interval mathematics, and theory of rough sets. However, all of these theories have their own difficulties which have been pointed out in [10]. Maji et al. [9] and Molodtsov [10] suggested that one reason for these difficulties may be due to the inadequacy of the parametrization tool of the theory. To overcome these difficulties, Molodtsov [10] introduced the concept of soft set as a new mathematical tool for dealing with uncertainties that is free from the difficulties that have troubled the usual theoretical approaches. Molodtsov pointed out several directions for the applications of soft sets. The algebraic structure of set theories dealing with uncertainties has been studied by some authors. The most appropriate theory for dealing with uncertainties

Received February 1, 2010.

2010 Mathematics Subject Classification. 20N25, 08A72.

Key words and phrases. $(\epsilon, \in)$-fuzzy subgroup, (strong) $\left(\in, \in \vee \mathrm{q}_{k}\right)$-fuzzy subgroup, $(\in$, $\left.\in \vee \mathrm{q}_{k}\right)$-fuzzy subgroup generated by a fuzzy subset, $\left(\in, \in \vee \mathrm{q}_{k}\right)$-fuzzy normal subgroup, $(\in$, $\in \vee \mathrm{q}_{k}$ )-fuzzy left (resp. right) coset, $\left(\in \vee \mathrm{q}_{k}\right)$-level subgroup. 
is the theory of fuzzy sets developed by Zadeh [17]. Murali [13] proposed a definition of a fuzzy point belonging to fuzzy subset under a natural equivalence on fuzzy subset. The idea of quasi-coincidence of a fuzzy point with a fuzzy set, which is mentioned in [14], played a vital role to generate some different types of fuzzy subsets. It is worth pointing out that Bhakat and Das $[3,4]$ initiated the concepts of $(\alpha, \beta)$-fuzzy subgroups by using the "belongs to" relation $(\epsilon)$ and "quasi-coincident with" relation $(q)$ between a fuzzy point and a fuzzy subgroup, and introduced the concept of an $(\in, \in \vee q)$-fuzzy subgroup. In particular, an $(\in, \in \vee \mathrm{q})$-fuzzy subgroup is an important and useful generalization of Rosenfeld's fuzzy subgroup. Liu [8] defined the fuzzy normality of a fuzzy subgroup in 1982. A coherent study of the fuzzy normal subgroups was initiated by Mukherjee and Bhattacharya [11, 12]. This notion was further studied in detail by Bhakat [1, 2], Bhakat and Das [3], and Yuan et al. [16]. It is now natural to investigate similar type of generalizations of the existing subsystems with other algebraic structures, and to discuss more general types than well-known types. With this objective in view, we use a general form of quasi-coincidence with a fuzzy subset. We introduce the notion of (strong) $\left(\in, \in \vee q_{k}\right)$-fuzzy subgroup which is a generalization of an $(\in, \in \vee \mathrm{q})$-fuzzy subgroup, and the concept of an $\left(\in, \in \vee q_{k}\right)$-fuzzy normal subgroup. We give characterizations of an $\left(\in, \in \vee \mathrm{q}_{k}\right)$-fuzzy (normal) subgroup, and deal with several related properties. Using a chain of subgroups of a group, we make an $(\in$, $\left.\in \vee q_{k}\right)$-fuzzy subgroup. Using a fuzzy subset of a group, we generate an $(\in$, $\left.\in \vee \mathrm{q}_{k}\right)$-fuzzy subgroup. We introduce the notion of $\left(\in, \in \vee \mathrm{q}_{k}\right)$-fuzzy left (resp. right) coset, and investigate several properties. The important achievement of the study with an $\left(\in, \in \vee q_{k}\right)$-fuzzy (normal) subgroup is that the notion of an $\left(\in, \in \vee q\right.$ )-fuzzy (normal) subgroup is a special case of an $\left(\in, \in \vee q_{k}\right)$-fuzzy (normal) subgroup. Since the transition from non-membership to membership is gradual rather than abrupt in fuzzy set theory, Dubois and Prade [6] introduced a new concept in fuzzy set theory, that of a gradual element. The notion of gradual elements is a new concept which is dealt with in fuzzy set theory. Of course, it is worth us wile to study the relationship between the results in Dubois and Prade's paper [6] and the results in this paper in the near future.

\section{Preliminaries}

A fuzzy subset $\mathcal{A}$ of a group $G$ is called a fuzzy subgroup of $G$ ([15]) if it satisfies:

(a1) $(\forall x, y \in G)(\mathcal{A}(x y) \geq \min \{\mathcal{A}(x), \mathcal{A}(y)\})$,

(a2) $(\forall x \in G)\left(\mathcal{A}\left(x^{-1}\right) \geq \mathcal{A}(x)\right)$.

For any fuzzy subset $\mathcal{A}$ of a set $X$ and any $t \in[0,1]$ the set

$$
\mathcal{A}_{t}=\{x \in X \mid \mathcal{A}(x) \geq t\}
$$

is called an $\in$-level subset of $\mathcal{A}$. 
A fuzzy subset $\mathcal{A}$ of a set $X$ of the form

$$
\mathcal{A}(y):= \begin{cases}t \in(0,1] & \text { if } y=x \\ 0 & \text { if } y \neq x\end{cases}
$$

is said to be a fuzzy point with support $x$ and value $t$ and is denoted by $[x ; t]$.

For a fuzzy subset $\mathcal{A}$ of a set $X$, we say that a fuzzy point $[x ; t]$ is

(a3) contained in $\mathcal{A}$, denoted by $[x ; t] \in \mathcal{A}$, ([14]) if $\mathcal{A}(x) \geq t$.

(a4) quasi-coincident with $\mathcal{A}$, denoted by $[x ; t] \mathrm{q} \mathcal{A}$, ([14]) if $\mathcal{A}(x)+t>1$.

For a fuzzy point $[x ; t]$ and a fuzzy subset $\mathcal{A}$ of a set $X$, we say that

(a5) $[x ; t] \in \vee \mathrm{q} \mathcal{A}$ if $[x ; t] \in \mathcal{A}$ or $[x ; t] \mathrm{q} \mathcal{A}$.

(a6) $[x ; t] \bar{\alpha} \mathcal{A}$ if $[x ; t] \alpha \mathcal{A}$ does not hold for $\alpha \in\{\in, \mathrm{q}, \in \vee \mathrm{q}\}$.

Definition 2.1. A fuzzy subset $\mathcal{A}$ of a group $G$ is called an $(\in, \in)$-fuzzy subgroup of $G$ if for any $x, y \in G$ and $t, r \in(0,1]$,

(a7) $[x ; t] \in \mathcal{A},[y ; r] \in \mathcal{A} \Rightarrow[x y ; \min \{t, r\}] \in \mathcal{A}$.

(a8) $[x ; t] \in \mathcal{A} \Rightarrow\left[x^{-1} ; t\right] \in \mathcal{A}$.

Note that the notion of $(\in, \in)$-fuzzy subgroup coincide with the notion of fuzzy subgroup. Hence we have the following theorem.

Theorem 2.2. For any fuzzy subset $\mathcal{A}$ of a group $G$, the following are equivalent.

(1) $\mathcal{A}$ is an $(\in, \in)$-fuzzy subgroup of $G$.

(2) $(\forall t \in(0,1])\left(\mathcal{A}_{t} \neq \emptyset \Rightarrow \mathcal{A}_{t}\right.$ is a subgroup of $\left.G\right)$.

\section{Generalizations of $(\in, \in \vee$ q)-level subsets}

Let $t \in(0,1]$ and $k \in[0,1)$. For a fuzzy point $[x ; t]$ and a fuzzy subset $\mathcal{A}$ of a set $G$, we say that

(b1) $[x ; t] \mathrm{q}_{k} \mathcal{A}$ if $\mathcal{A}(x)+t+k>1$.

(b2) $[x ; t] \in \vee \mathrm{q}_{k} \mathcal{A}$ if $[x ; t] \in \mathcal{A}$ or $[x ; t] \mathrm{q}_{k} \mathcal{A}$.

(b3) $[x ; t] \underline{\mathrm{q}} \mathcal{A}$ if $\mathcal{A}(x)+t \geq 1$.

(b4) $[x ; t] \underline{\mathrm{q}}_{k} \mathcal{A}$ if $\mathcal{A}(x)+t+k \geq 1$.

(b5) $[x ; t] \bar{\alpha} \mathcal{A}$ if $[x ; t] \alpha \mathcal{A}$ does not hold for $\alpha \in\left\{\mathrm{q}_{k}, \in \vee \mathrm{q}_{k}, \underline{\mathrm{q}}, \underline{\mathrm{q}}_{k}\right\}$.

Definition 3.1. Let $\mathcal{A}$ be a fuzzy subset of a set $G$ and $t \in(0,1]$. Then the set

$$
Q(\mathcal{A} ; t):=\{x \in X \mid[x ; t] \mathrm{q} \mathcal{A}\}
$$

is called the q-level subset of $G$, the set

$$
Q_{k}(\mathcal{A} ; t):=\left\{x \in X \mid[x ; t] \mathrm{q}_{k} \mathcal{A}\right\}
$$

is called the $\mathrm{q}_{\mathrm{k}}$-level subset of $G$, the set

$$
\underline{Q}(\mathcal{A} ; t):=\{x \in X \mid[x ; t] \underline{\mathrm{q}} \mathcal{A}\}
$$

is called the closed q-level subset of $G$, the set

$$
\underline{Q}_{k}(\mathcal{A} ; t):=\left\{x \in X \mid[x ; t] \underline{\mathrm{q}}_{k} \mathcal{A}\right\}
$$


is called the closed $\mathrm{q}_{k}$-level subset of $G$, the set

$$
U(\mathcal{A} ; t):=\{x \in G \mid[x ; t] \in \vee \mathrm{q} \mathcal{A}\}=\mathcal{A}_{t} \cup Q(\mathcal{A} ; t)
$$

is called $(\in \vee \mathrm{q})$-level subset of $G$ (see [1]), the set

$$
U_{k}(\mathcal{A} ; t):=\left\{x \in G \mid[x ; t] \in \vee \mathrm{q}_{k} \mathcal{A}\right\}=\mathcal{A}_{t} \cup Q_{k}(\mathcal{A} ; t)
$$

is called an $\left(\in \vee \mathrm{q}_{k}\right)$-level subset of $G$, and the set

$$
\underline{U}_{k}(\mathcal{A} ; t):=\left\{x \in G \mid[x ; t] \in \vee \underline{q}_{k} \mathcal{A}\right\}=\mathcal{A}_{t} \cup \underline{Q}_{k}(\mathcal{A} ; t)
$$

is called a closed $\left(\in \vee \mathrm{q}_{k}\right)$-level subset of $G$.

Note that $\mathcal{A}_{t} \subseteq U(\mathcal{A} ; t) \subseteq U_{k}(\mathcal{A} ; t)$ for any $t \in(0,1]$ and $k \in[0,1)$. However, the reverse inclusions may not be true.

Example 3.2. Let $\mathcal{A}$ be a fuzzy subset of a set $G=\{a, b, c, d, e, f\}$ defined by

$$
\mathcal{A}=\left(\begin{array}{cccccc}
a & b & c & d & e & f \\
0.6 & 0.3 & 0.8 & 0.2 & 0.5 & 0.6
\end{array}\right) .
$$

Then $\mathcal{A}_{0.55}=\{a, c, f\} \neq U(\mathcal{A} ; 0.55)=\{a, c, e, f\} \neq U_{0.23}(\mathcal{A} ; 0.55)=\{a, b, c, e, f\}$.

Proposition 3.3. Let $\mathcal{A}$ be a fuzzy subset of a set $G$. For any $m, n \in[0,1)$, we have

$$
m<n \Rightarrow(\forall t \in(0,1])\left(U_{m}(\mathcal{A} ; t) \subseteq U_{n}(\mathcal{A} ; t)\right) .
$$

Proof. Straightforward.

If $t>r$, then $U_{k}(\mathcal{A} ; t)$ may not be a subset of $U_{k}(\mathcal{A} ; r)$ for some $k \in[0,1)$. In Example 3.2, $b \in U_{0.1}(\mathcal{A} ; 0.7)$ but $b \notin U_{0.1}(\mathcal{A} ; 0.47)$.

Proposition 3.4. Let $\mathcal{A}$ and $\mathcal{B}$ be fuzzy subsets of a set $G$. Then

(1) $U_{k}(\mathcal{A} \cup \mathcal{B} ; t)=U_{k}(\mathcal{A} ; t) \cup U_{k}(\mathcal{B} ; t)$.

(2) $U_{k}(\mathcal{A} \cap \mathcal{B} ; t)=U_{k}(\mathcal{A} ; t) \cap U_{k}(\mathcal{B} ; t)$.

(3) $U_{k}(\mathcal{A} \cup(\mathcal{B} \cap \mathcal{C}) ; t)=U_{k}(\mathcal{A} \cup \mathcal{B} ; t) \cap U_{k}(\mathcal{A} \cup \mathcal{C} ; t)$

(4) $U_{k}(\mathcal{A} \cap(\mathcal{B} \cup \mathcal{C}) ; t)=U_{k}(\mathcal{A} \cap \mathcal{B} ; t) \cup U_{k}(\mathcal{A} \cap \mathcal{C} ; t)$.

Proof. We have

$$
\begin{aligned}
x \in U_{k}(\mathcal{A} \cup \mathcal{B} ; t) \Leftrightarrow & {[x ; t] \in \vee \mathrm{q}_{k}(\mathcal{A} \cup \mathcal{B}) } \\
\Leftrightarrow & (\mathcal{A} \cup \mathcal{B})(x) \geq t \text { or }(\mathcal{A} \cup \mathcal{B})(x)+t>1-k \\
\Leftrightarrow & {[\mathcal{A}(x) \geq t \text { or } \mathcal{B}(x) \geq t] \text { or } } \\
& {[\mathcal{A}(x)+t>1-k \text { or } \mathcal{B}(x)+t>1-k] } \\
\Leftrightarrow & {[\mathcal{A}(x) \geq t \text { or } \mathcal{A}(x)+t>1-k] \text { or } } \\
& {[\mathcal{B}(x) \geq t \text { or } \mathcal{B}(x)+t>1-k] } \\
\Leftrightarrow & {[x ; t] \in \vee \mathrm{q}_{k} \mathcal{A} \text { or }[x ; t] \in \vee \mathrm{q}_{k} \mathcal{B} } \\
\Leftrightarrow & x \in U_{k}(\mathcal{A} ; t) \text { or } x \in U_{k}(\mathcal{B} ; t) \\
\Leftrightarrow & x \in U_{k}(\mathcal{A} ; t) \cup U_{k}(\mathcal{B} ; t)
\end{aligned}
$$


and

$$
\begin{aligned}
x \in U_{k}(\mathcal{A} \cap \mathcal{B} ; t) \Leftrightarrow & {[x ; t] \in \vee \mathrm{q}_{k}(\mathcal{A} \cap \mathcal{B}) } \\
\Leftrightarrow & (\mathcal{A} \cap \mathcal{B})(x) \geq t \text { or }(\mathcal{A} \cap \mathcal{B})(x)+t>1-k \\
\Leftrightarrow & {[\mathcal{A}(x) \geq t \text { and } \mathcal{B}(x) \geq t] \text { or } } \\
& {[\mathcal{A}(x)+t>1-k \text { and } \mathcal{B}(x)+t>1-k] } \\
\Leftrightarrow & {[\mathcal{A}(x) \geq t \text { or } \mathcal{A}(x)+t>1-k] \text { and } } \\
& {[\mathcal{B}(x) \geq t \text { or } \mathcal{B}(x)+t>1-k] } \\
\Leftrightarrow & {[x ; t] \in \vee \mathrm{q}_{k} \mathcal{A} \text { and }[x ; t] \in \vee \mathrm{q}_{k} \mathcal{B} } \\
\Leftrightarrow & x \in U_{k}(\mathcal{A} ; t) \text { and } x \in U_{k}(\mathcal{B} ; t) \\
\Leftrightarrow & x \in U_{k}(\mathcal{A} ; t) \cap U_{k}(\mathcal{B} ; t)
\end{aligned}
$$

which proves (1) and (2). Using (1) and (2), one can show that (3) and (4).

If we take $k=0$ in Proposition 3.4, then we have the following corollary.

Corollary 3.5 ([1]). Let $\mathcal{A}$ and $\mathcal{B}$ be fuzzy subsets of a set $G$. Then

(1) $U(\mathcal{A} \cup \mathcal{B} ; t)=U(\mathcal{A} ; t) \cup U(\mathcal{B} ; t)$.

(2) $U(\mathcal{A} \cap \mathcal{B} ; t)=U(\mathcal{A} ; t) \cap U(\mathcal{B} ; t)$.

(3) $U(\mathcal{A} \cup(\mathcal{B} \cap \mathcal{C}) ; t)=U(\mathcal{A} \cup \mathcal{B} ; t) \cap U(\mathcal{A} \cup \mathcal{C} ; t)$.

(4) $U(\mathcal{A} \cap(\mathcal{B} \cup \mathcal{C}) ; t)=U(\mathcal{A} \cap \mathcal{B} ; t) \cup U(\mathcal{A} \cap \mathcal{C} ; t)$.

Proposition 3.6. For a fuzzy subset $\mathcal{A}$ of a set $G$, we have

$$
U_{k}(\mathcal{A} ; t)^{c} \subseteq \mathcal{A}_{1-t}^{c} \cap \mathcal{A}_{t+k}^{c},
$$

where $\mathcal{A}^{c}$ denotes the complement of $\mathcal{A}$, that is, $\mathcal{A}^{c}(x)=1-\mathcal{A}(x)$ for all $x \in G$.

Proof. We get

$$
\begin{aligned}
x \in U_{k}(\mathcal{A} ; t)^{c} & \Rightarrow x \notin U_{k}(\mathcal{A} ; t) \Rightarrow[x ; t] \overline{\in \vee \mathrm{q}_{k}} \mathcal{A} \\
& \Rightarrow \quad[x ; t] \bar{\in} \mathcal{A} \text { and }[x ; t] \overline{\mathrm{q}_{k}} \mathcal{A} \\
& \Rightarrow \mathcal{A}(x)<t \text { and } \mathcal{A}(x)+t+k \leq 1 \\
& \Rightarrow \mathcal{A}^{c}(x)=1-\mathcal{A}(x)>1-t \text { and } \\
& \mathcal{A}^{c}(x)=1-\mathcal{A}(x) \geq t+k \\
\Rightarrow & x \in \mathcal{A}_{1-t}^{c} \text { and } x \in \mathcal{A}_{t+k}^{c} \\
\Rightarrow & x \in \mathcal{A}_{1-t}^{c} \cap \mathcal{A}_{t+k}^{c},
\end{aligned}
$$

and so $U_{k}(\mathcal{A} ; t)^{c} \subseteq \mathcal{A}_{1-t}^{c} \cap \mathcal{A}_{t+k}^{c}$.

Proposition 3.7. For fuzzy subsets $\mathcal{A}$ and $\mathcal{B}$ of a set $G$, we have

$$
\left(U_{k}(\mathcal{A} ; t) \cup U_{k}(\mathcal{B} ; t)\right)^{c} \subseteq \mathcal{A}_{1-t}^{c} \cap \mathcal{B}_{1-t}^{c} \cap \mathcal{A}_{t+k}^{c} \cap \mathcal{B}_{t+k}^{c} .
$$

Proof. It follows from Propositions 3.4(1) and 3.6. 
TABLE 1. Multiplication table for $G$

\begin{tabular}{l|lll}
\hline & $a$ & $b$ & $c$ \\
\hline$a$ & $a$ & $b$ & $c$ \\
$b$ & $b$ & $c$ & $a$ \\
$c$ & $c$ & $a$ & $b$ \\
\hline
\end{tabular}

\section{Generalizations of $(\in, \in \vee$ q)-fuzzy subgroups}

In what follows, let $G$ denote a group with $e$ as the identity element, and $k$ an arbitrary element of $[0,1)$ unless otherwise specified.

Definition 4.1. A fuzzy subset $\mathcal{A}$ of $G$ is called a strong $\left(\in, \in \vee \mathrm{q}_{k}\right)$-fuzzy subgroup of $G$ if for any $x, y \in G$ and $t, r \in(0,1]$,

(c1) $[x ; t] \in \mathcal{A},[y ; r] \in \mathcal{A} \Rightarrow[x y ; \min \{t, r\}] \in \vee \mathrm{q}_{k} \mathcal{A}$.

(c2) $\mathcal{A}\left(x^{-1}\right) \geq \mathcal{A}(x)$.

A strong $\left(\in, \in \vee \mathrm{q}_{k}\right)$-fuzzy subgroup of $G$ with $k=0$ is called a strong $(\in, \in \vee \mathrm{q})$-fuzzy subgroup of $G$.

Lemma 4.2. The condition (c2) is equivalent to the following condition

(c3) $(\forall x \in G)(\forall t \in(0,1])\left([x ; t] \in \mathcal{A} \Rightarrow\left[x^{-1} ; t\right] \in \mathcal{A}\right)$.

Proof. Straightforward.

Definition 4.3. A fuzzy subset $\mathcal{A}$ of $G$ is called an $\left(\in, \in \vee \mathrm{q}_{k}\right)$-fuzzy subgroup of $G$ if it satisfies (c1) and

(c4) $(\forall x \in G)(\forall t \in(0,1])\left([x ; t] \in \mathcal{A} \Rightarrow\left[x^{-1} ; t\right] \in \vee \mathrm{q}_{k} \mathcal{A}\right)$

An $\left(\in, \in \vee \mathrm{q}_{k}\right)$-fuzzy subgroup of $G$ with $k=0$ is called an $(\in, \in \vee \mathrm{q})$ fuzzy subgroup of $G$. Obviously, every strong $\left(\in, \in \vee \mathrm{q}_{k}\right)$-fuzzy subgroup is an $\left(\in, \in \vee \mathrm{q}_{k}\right)$-fuzzy subgroup, but the converse is not true in general as seen in the following example.

Example 4.4. Let $G=\{a, b, c\}$ be the cyclic group where the multiplication is defined by Table 1 . Let $\mathcal{A}$ be a fuzzy subset of $G$ defined by $\mathcal{A}(a)=0.4$, $\mathcal{A}(b)=0.7$ and $\mathcal{A}(c)=0.9$. Then $\mathcal{A}$ is an $\left(\in, \in \vee \mathrm{q}_{k}\right)$-fuzzy subgroup of $G$ for $k \in[0.2,1)$. But it is not a strong $\left(\in, \in \vee \mathrm{q}_{k}\right)$-fuzzy subgroup of $G$ since

$$
\mathcal{A}\left(c^{-1}\right)=\mathcal{A}(b)=0.7 \ngtr 0.9=\mathcal{A}(c) .
$$

Theorem 4.5. A fuzzy subset $\mathcal{A}$ of $G$ is an $\left(\in, \in \vee \mathrm{q}_{k}\right)$-fuzzy subgroup of $G$ if and only if it satisfies:

(1) $(\forall x, y \in G)\left(\mathcal{A}(x y) \geq \min \left\{\mathcal{A}(x), \mathcal{A}(y), \frac{1-k}{2}\right\}\right)$,

(2) $(\forall x \in G)\left(\mathcal{A}\left(x^{-1}\right) \geq \min \left\{\mathcal{A}(x), \frac{1-k}{2}\right\}\right)$. 
Proof. Suppose that $\mathcal{A}$ is an $\left(\in, \in \vee \mathrm{q}_{k}\right)$-fuzzy subgroup of $G$. Let $x \in G$ and assume that $\mathcal{A}(x)<\frac{1-k}{2}$. If $\mathcal{A}\left(x^{-1}\right)<\mathcal{A}(x)$, then $\mathcal{A}\left(x^{-1}\right)<t \leq \mathcal{A}(x)$ for some $t \in\left(0, \frac{1-k}{2}\right)$. It follows that $[x ; t] \in \mathcal{A}$ but $\left[x^{-1} ; t\right] \bar{\in} \mathcal{A}$. Since $\mathcal{A}\left(x^{-1}\right)+t<2 t<$ $1-k$, we get $\left[x^{-1} ; t\right] \overline{\mathrm{q}_{k}} \mathcal{A}$. Therefore $\left[x^{-1} ; t\right] \in \vee \mathrm{q}_{k} \mathcal{A}$, which is a contradiction. Hence $\mathcal{A}\left(x^{-1}\right) \geq \mathcal{A}(x)$. Now, if $\mathcal{A}(x) \geq \frac{1-k}{2}$, then $\left[x ; \frac{1-k}{2}\right] \in \mathcal{A}$ and so $\left[x^{-1} ; \frac{1-k}{2}\right] \in \vee \mathrm{q}_{k} \mathcal{A}$ by $(\mathrm{c} 4)$. Hence $\mathcal{A}\left(x^{-1}\right) \geq \frac{1-k}{2}$ or $\mathcal{A}\left(x^{-1}\right)+\frac{1-k}{2}>1-k$. It follows that $\mathcal{A}\left(x^{-1}\right) \geq \frac{1-k}{2}$. Otherwise, $\mathcal{A}\left(x^{-1}\right)+\frac{1-k}{2}<\frac{1-k}{2}+\frac{1-k}{2}=$ $1-k$, a contradiction. Consequently, $\mathcal{A}\left(x^{-1}\right) \geq \min \left\{\mathcal{A}(x), \frac{1-k}{2}\right\}$ for all $x \in$ $G$. Let $x, y \in G$ and suppose that $\min \{\mathcal{A}(x), \mathcal{A}(y)\}<\frac{1-k}{2}$. We claim that $\mathcal{A}(x y) \geq \min \{\mathcal{A}(x), \mathcal{A}(y)\}$. If not, then $\mathcal{A}(x y)<t \leq \min \{\mathcal{A}(x), \mathcal{A}(y)\}$ for some $t \in\left(0, \frac{1-k}{2}\right)$. It follows that $[x ; t] \in \mathcal{A}$ and $[y ; t] \in \mathcal{A}$, but $[x y ; t] \bar{\in} \mathcal{A}$ and $\mathcal{A}(x y)+t<2 t<1-k$, i.e., $[x y ; t] \overline{\mathrm{q}} k \mathcal{A}$. This is a contradiction, and so $\mathcal{A}(x y) \geq$ $\min \{\mathcal{A}(x), \mathcal{A}(y)\}$ whenever $\min \{\mathcal{A}(x), \mathcal{A}(y)\}<\frac{1-k}{2}$. If $\min \{\mathcal{A}(x), \mathcal{A}(y)\} \geq$ $\frac{1-k}{2}$, then $\left[x ; \frac{1-k}{2}\right] \in \mathcal{A}$ and $\left[y ; \frac{1-k}{2}\right] \in \mathcal{A}$. Since $\mathcal{A}$ is an $\left(\in, \in \vee \mathrm{q}_{k}\right)$-fuzzy subgroup, it follows from (c1) that

$$
\left[x y ; \frac{1-k}{2}\right]=\left[x y ; \min \left\{\frac{1-k}{2}, \frac{1-k}{2}\right\}\right] \in \vee \mathrm{q}_{k} \mathcal{A}
$$

so that $\mathcal{A}(x y) \geq \frac{1-k}{2}$ or $\mathcal{A}(x y)+\frac{1-k}{2}>1-k$. If $\mathcal{A}(x y)<\frac{1-k}{2}$, then

$$
\mathcal{A}(x y)+\frac{1-k}{2}<\frac{1-k}{2}+\frac{1-k}{2}=1-k,
$$

i.e., $\left[x y ; \frac{1-k}{2}\right] \overline{\mathrm{q}_{k}} \mathcal{A}$. This is impossible, and thus $\mathcal{A}(x y) \geq \frac{1-k}{2}$. Consequently, $\mathcal{A}(x y) \geq \min \left\{\mathcal{A}(x), \mathcal{A}(y), \frac{1-k}{2}\right\}$ for all $x, y \in G$.

Conversely, assume that (1) and (2) are valid. Let $x, y \in G$ and $t, r \in(0,1]$ be such that $[x ; t] \in \mathcal{A}$ and $[y ; r] \in \mathcal{A}$. Then $\mathcal{A}(x) \geq t$ and $\mathcal{A}(y) \geq r$. Suppose that $[x y ; \min \{t, r\}] \bar{\epsilon}$, i.e., $\mathcal{A}(x y)<\min \{t, r\}$. If $\min \{\mathcal{A}(x), \mathcal{A}(y)\}<\frac{1-k}{2}$, then

$$
\mathcal{A}(x y) \geq \min \left\{\mathcal{A}(x), \mathcal{A}(y), \frac{1-k}{2}\right\}=\min \{\mathcal{A}(x), \mathcal{A}(y)\} \geq \min \{t, r\}
$$

which is a contradiction. Hence $\min \{\mathcal{A}(x), \mathcal{A}(y)\} \geq \frac{1-k}{2}$, and so

$$
\mathcal{A}(x y)+\min \{t, r\}>2 \mathcal{A}(x y) \geq 2 \min \left\{\mathcal{A}(x), \mathcal{A}(y), \frac{1-k}{2}\right\}=1-k,
$$

i.e., $[x y ; \min \{t, r\}] \mathrm{q}_{k} \mathcal{A}$. Therefore $[x y ; \min \{t, r\}] \in \vee \mathrm{q}_{k} \mathcal{A}$. Now let $x \in G$ and $t \in(0,1]$ be such that $[x ; t] \in \mathcal{A}$. Then $\mathcal{A}(x) \geq t$. Assume that $\mathcal{A}\left(x^{-1}\right)<t$. If $\mathcal{A}(x)<\frac{1-k}{2}$, then $\mathcal{A}\left(x^{-1}\right) \geq \min \left\{\mathcal{A}(x), \frac{1-k}{2}\right\}=\mathcal{A}(x) \geq t$, a contradiction. Thus $\mathcal{A}(x) \geq \frac{1-k}{2}$, and so

$$
\mathcal{A}\left(x^{-1}\right)+t>2 \mathcal{A}\left(x^{-1}\right) \geq 2 \min \left\{\mathcal{A}(x), \frac{1-k}{2}\right\}=1-k .
$$

Hence $\left[x^{-1} ; t\right] \in \vee \mathrm{q}_{k} \mathcal{A}$. Consequently, $\mathcal{A}$ is an $\left(\in, \in \vee \mathrm{q}_{k}\right)$-fuzzy subgroup of $G$.

Corollary 4.6 ([4]). A fuzzy subset $\mathcal{A}$ of $G$ is an $(\in, \in \vee \mathrm{q})$-fuzzy subgroup of $G$ if and only if it satisfies:

(1) $(\forall x, y \in G)(\mathcal{A}(x y) \geq \min \{\mathcal{A}(x), \mathcal{A}(y), 0.5\})$,

(2) $(\forall x \in G)\left(\mathcal{A}\left(x^{-1}\right) \geq \min \{\mathcal{A}(x), 0.5\}\right)$. 
Theorem 4.7. For a fuzzy subset $\mathcal{A}$ of $G$, the following assertions are equivalent:

(1) $\left(\forall t \in\left(\frac{1-k}{2}, 1\right]\right)\left(\mathcal{A}_{t} \neq \emptyset \Rightarrow \mathcal{A}_{t}\right.$ is a subgroup of $\left.G\right)$.

(2) $\mathcal{A}$ satisfies the following conditions:

(2.1) $(\forall x, y \in G)\left(\max \left\{\mathcal{A}(x y), \frac{1-k}{2}\right\} \geq \min \{\mathcal{A}(x), \mathcal{A}(y)\}\right)$,

(2.2) $(\forall x, y \in G)\left(\max \left\{\mathcal{A}\left(x^{-1}\right), \frac{1-k}{2}\right\} \geq \mathcal{A}(x)\right)$.

Proof. Assume that (1) is valid. If there exist $a, b \in G$ such that

$$
\max \left\{\mathcal{A}(a b), \frac{1-k}{2}\right\}<\min \{\mathcal{A}(a), \mathcal{A}(b)\},
$$

then $\max \left\{\mathcal{A}(a b), \frac{1-k}{2}\right\}<t \leq \min \{\mathcal{A}(a), \mathcal{A}(b)\}$ where $t \in\left(\frac{1-k}{2}, 1\right]$. Then $a, b \in$ $\mathcal{A}_{t}$ and so $a b \in \mathcal{A}_{t}$ since $\mathcal{A}_{t}$ is a subgroup of $G$. It follows that $\mathcal{A}(a b) \geq t$, a contradiction. Therefore $\max \left\{\mathcal{A}(x y), \frac{1-k}{2}\right\} \geq \min \{\mathcal{A}(x), \mathcal{A}(y)\}$ for all $x, y \in G$. Suppose that (2.2) is not valid. Then

$$
\max \left\{\mathcal{A}\left(a^{-1}\right), \frac{1-k}{2}\right\}<t_{a} \leq \mathcal{A}(a)
$$

for some $a \in G$, where $t_{a} \in\left(\frac{1-k}{2}, 1\right]$. Hence $a \in \mathcal{A}_{t_{a}}$ but $a^{-1} \notin \mathcal{A}_{t_{a}}$ which is a contradiction. Thus $\max \left\{\mathcal{A}\left(x^{-1}\right), \frac{1-k}{2}\right\} \geq \mathcal{A}(x)$ for all $x \in G$.

Conversely, assume that $\mathcal{A}$ satisfies two conditions (2.1) and (2.2). Let $t \in\left(\frac{1-k}{2}, 1\right]$ such that $\mathcal{A}_{t} \neq \emptyset$. Let $x \in \mathcal{A}_{t}$. Then $\mathcal{A}(x) \geq t$ and so

$$
\max \left\{\mathcal{A}\left(x^{-1}\right), \frac{1-k}{2}\right\} \geq \mathcal{A}(x) \geq t
$$

by (2.2). Since $t \in\left(\frac{1-k}{2}, 1\right]$, it follows that $\mathcal{A}\left(x^{-1}\right) \geq t$ so that $x^{-1} \in \mathcal{A}_{t}$. Let $x, y \in \mathcal{A}_{t}$. Using (2.1), we have

$$
\max \left\{\mathcal{A}(x y), \frac{1-k}{2}\right\} \geq \min \{\mathcal{A}(x), \mathcal{A}(y)\} \geq t>\frac{1-k}{2},
$$

and thus $\mathcal{A}(x y) \geq t$, i.e., $x y \in \mathcal{A}_{t}$. Consequently, $\mathcal{A}_{t}$ is a subgroup of $G$ for all $t \in\left(\frac{1-k}{2}, 1\right]$.

Corollary 4.8 ([16]). Let $\mathcal{A}$ be a fuzzy subset of $G$. Then $\mathcal{A}_{t}$ is a subgroup of $G$ for all $t \in(0.5,1]$ if and only if it satisfies:

(1) $(\forall x, y \in G)(\max \{\mathcal{A}(x y), 0.5\} \geq \min \{\mathcal{A}(x), \mathcal{A}(y)\})$,

(2) $(\forall x, y \in G)\left(\max \left\{\mathcal{A}\left(x^{-1}\right), 0.5\right\} \geq \mathcal{A}(x)\right)$.

If $\mathcal{A}$ is an $\left(\epsilon, \in \vee \mathrm{q}_{k}\right)$-fuzzy subgroup of $G$, then for any $t \in(0,1], \mathcal{A}_{t}$ may not be a subgroup of $G$ as seen in the following example.

Example 4.9. Consider the Klein's 4-group $G=\{e, a, b, c\}$ where the multiplication is defined by Table 2 . Let $\mathcal{A}$ be a fuzzy subset of $G$ defined by

$$
\mathcal{A}=\left(\begin{array}{cccc}
e & a & b & c \\
0.35 & 0.7 & 0.2 & 0.2
\end{array}\right) \text {. }
$$

Then $\mathcal{A}$ is an $\left(\in, \in \vee \mathrm{q}_{k}\right)$-fuzzy subgroup of $G$ for $k=0.3$, but if $t>0.35$, then $\mathcal{A}_{t}=\{a\}$ which is not a subgroup of $G$ and hence we know that $\mathcal{A}$ is not an $(\in, \in)$-fuzzy subgroup of $G$.

We provide characterizations of an $\left(\in, \in \vee q_{k}\right)$-fuzzy subgroup. 
TABLE 2. Multiplication table for $G$

\begin{tabular}{l|llll}
\hline & $e$ & $a$ & $b$ & $c$ \\
\hline$e$ & $e$ & $a$ & $b$ & $c$ \\
$a$ & $a$ & $e$ & $c$ & $b$ \\
$b$ & $b$ & $c$ & $e$ & $a$ \\
$c$ & $c$ & $b$ & $a$ & $e$ \\
\hline
\end{tabular}

Theorem 4.10. For any fuzzy subset $\mathcal{A}$ of $G$, the following are equivalent:

(1) $\mathcal{A}$ is an $\left(\in, \in \vee \mathrm{q}_{k}\right)$-fuzzy subgroup of $G$.

(2) $\left(\forall t \in\left(0, \frac{1-k}{2}\right]\right)\left(\mathcal{A}_{t} \neq \emptyset \Rightarrow \mathcal{A}_{t}\right.$ is a subgroup of $\left.G\right)$.

We say that $\mathcal{A}_{t}$ is an $\left(\in \vee \mathrm{q}_{k}\right)$-level subgroup of $\mathcal{A}$ in $G$.

Proof. Assume that $\mathcal{A}$ is an $\left(\in, \in \vee \mathrm{q}_{k}\right)$-fuzzy subgroup of $G$ and let $t \in\left(0, \frac{1-k}{2}\right]$ such that $\mathcal{A}_{t} \neq \emptyset$. Let $x, y \in \mathcal{A}_{t}$. Using Theorem 4.5, we have

$$
\mathcal{A}(x y) \geq \min \left\{\mathcal{A}(x), \mathcal{A}(y), \frac{1-k}{2}\right\} \geq \min \left\{t, \frac{1-k}{2}\right\}=t
$$

and

$$
\mathcal{A}\left(x^{-1}\right) \geq \min \left\{\mathcal{A}(x), \frac{1-k}{2}\right\} \geq \min \left\{t, \frac{1-k}{2}\right\}=t .
$$

Hence $x y \in \mathcal{A}_{t}$ and $x^{-1} \in \mathcal{A}_{t}$, i.e., $\mathcal{A}_{t}$ is a subgroup of $G$.

Conversely, suppose that (2) is valid. If there exist $a, b \in G$ such that

$$
\mathcal{A}(a b)<\min \left\{\mathcal{A}(a), \mathcal{A}(b), \frac{1-k}{2}\right\},
$$

then $\mathcal{A}(a b)<t \leq \min \left\{\mathcal{A}(a), \mathcal{A}(b), \frac{1-k}{2}\right\}$ for some $t \in(0,1]$. Then $t \leq \frac{1-k}{2}$ and so $t \in\left(0, \frac{1-k}{2}\right]$, and $a, b \in \mathcal{A}_{t}$. But $a b \notin \mathcal{A}_{t}$, which is a contradiction. Therefore

$$
\mathcal{A}(x y) \geq \min \left\{\mathcal{A}(x), \mathcal{A}(y), \frac{1-k}{2}\right\}
$$

for all $x, y \in G$. If there exists $a \in G$ such that $\mathcal{A}\left(a^{-1}\right)<\min \left\{\mathcal{A}(a), \frac{1-k}{2}\right\}$, then $\mathcal{A}\left(a^{-1}\right)<t_{a} \leq \min \left\{\mathcal{A}(a), \frac{1-k}{2}\right\}$ for some $t_{a} \in(0,1]$. Then $t_{a} \leq \frac{1-k}{2}$ and so $t_{a} \in\left(0, \frac{1-k}{2}\right]$, and $a \in \mathcal{A}_{t_{a}}$. But $a^{-1} \notin \mathcal{A}_{t_{a}}$. This is impossible. Therefore $\mathcal{A}\left(x^{-1}\right) \geq \min \left\{\mathcal{A}(x), \frac{1-k}{2}\right\}$ for all $x \in G$. Using Theorem 4.5, we conclude that $\mathcal{A}$ is an $\left(\in, \in \vee \mathrm{q}_{k}\right)$-fuzzy subgroup of $G$.

Corollary 4.11 ([4, Theorem 3.7]). For any fuzzy subset $\mathcal{A}$ of $G$, the following are equivalent:

(1) $\mathcal{A}$ is an $(\in, \in \vee \mathrm{q})$-fuzzy subgroup of $G$.

(2) $(\forall t \in(0,0.5])\left(\mathcal{A}_{t} \neq \emptyset \Rightarrow \mathcal{A}_{t}\right.$ is a subgroup of $\left.G\right)$.

Theorem 4.12. If $\mathcal{A}$ is an $\left(\in, \in \vee \mathrm{q}_{k}\right)$-fuzzy subgroup of $G$, then $\left(\forall t \in\left(\frac{1-k}{2}, 1\right]\right)\left(\underline{Q}_{k}(\mathcal{A} ; t) \neq \emptyset \Rightarrow \underline{Q}_{k}(\mathcal{A} ; t)\right.$ is a subgroup of $\left.G\right)$. 
Proof. Assume that $\underline{Q}_{k}(\mathcal{A} ; t) \neq \emptyset$ for $t \in\left(\frac{1-k}{2}, 1\right]$. Let $x, y \in \underline{Q}_{k}(\mathcal{A} ; t)$. Then $[x ; t] \underline{\mathrm{q}}_{k} \mathcal{A}$ and $[y ; t] \underline{\mathrm{q}}_{k} \mathcal{A}$, that is, $\mathcal{A}(x)+t \geq 1-k$ and $\mathcal{A}(y)+t \geq 1-k$. Using Theorem 4.5, we have

$$
\mathcal{A}(x y) \geq \min \left\{\mathcal{A}(x), \mathcal{A}(y), \frac{1-k}{2}\right\} \geq \min \left\{1-k-t, \frac{1-k}{2}\right\}
$$

and

$$
\mathcal{A}\left(x^{-1}\right) \geq \min \left\{\mathcal{A}(x), \frac{1-k}{2}\right\} \geq \min \left\{1-k-t, \frac{1-k}{2}\right\} .
$$

Since $t \in\left(\frac{1-k}{2}, 1\right]$, we have $1-k-t<\frac{1-k}{2}$. It follows that $\mathcal{A}(x y) \geq 1-k-t$ and $\mathcal{A}\left(x^{-1}\right) \geq 1-k-t$ so that $x y \in \underline{Q}_{k}(\mathcal{A} ; t)$ and $x^{-1} \in \underline{Q}_{k}(\mathcal{A} ; t)$. Hence $\underline{Q}_{k}(\mathcal{A} ; t)$ is a subgroup of $G$.

If we take $k=0$ in Theorem 4.12 , then we have the following corollary.

Corollary 4.13. If $\mathcal{A}$ is an $(\in, \in \vee \mathrm{q})$-fuzzy subgroup of $G$, then

$$
(\forall t \in(0.5,1])(\underline{Q}(\mathcal{A} ; t) \neq \emptyset \Rightarrow \underline{Q}(\mathcal{A} ; t) \text { is a subgroup of } G) .
$$

Theorem 4.14. For any fuzzy subset $\mathcal{A}$ of $G$, the following are equivalent:

(1) $\mathcal{A}$ is an $\left(\in, \in \vee \mathrm{q}_{k}\right)$-fuzzy subgroup of $G$.

(2) $(\forall t \in(0,1])\left(\underline{U}_{k}(\mathcal{A} ; t) \neq \emptyset \Rightarrow \underline{U}_{k}(\mathcal{A} ; t)\right.$ is a subgroup of $\left.G\right)$.

Proof. Assume that $\mathcal{A}$ is an $\left(\in, \in \vee \mathrm{q}_{k}\right)$-fuzzy subgroup of $G$ and let $t \in(0,1]$ such that $\underline{U}_{k}(\mathcal{A} ; t) \neq \emptyset$. Let $x \in \underline{U}_{k}(\mathcal{A} ; t)$. Then $\mathcal{A}(x) \geq t$ or $\mathcal{A}(x)+t \geq 1-k$. Using Theorem 4.5(2), we obtain

$$
\mathcal{A}\left(x^{-1}\right) \geq \min \left\{\mathcal{A}(x), \frac{1-k}{2}\right\} .
$$

We consider two cases: $\mathcal{A}(x) \leq \frac{1-k}{2}$ and $\mathcal{A}(x)>\frac{1-k}{2}$. For the first case, we have $\mathcal{A}\left(x^{-1}\right) \geq \mathcal{A}(x)$ by (4.1). Thus if $\mathcal{A}(x) \geq t$, then $\mathcal{A}\left(x^{-1}\right) \geq t$, and so $x^{-1} \in \mathcal{A}_{t} \subseteq \underline{U}_{k}(\mathcal{A} ; t)$. If $\mathcal{A}(x)+t \geq 1-k$, then $\mathcal{A}\left(x^{-1}\right)+t \geq \mathcal{A}(x)+t \geq 1-k$ which implies that $\left[x^{-1} ; t\right] \underline{\mathrm{q}}_{k} \mathcal{A}$, i.e., $x^{-1} \in \underline{Q}_{k}(\mathcal{A} ; t) \subseteq \underline{U}_{k}(\mathcal{A} ; t)$. Combining the second case and (4.1) induces $\mathcal{A}\left(x^{-1}\right) \geq \frac{1-k}{2}$. If $t \leq \frac{1-k}{2}$, then $\mathcal{A}\left(x^{-1}\right) \geq t$ and hence $x^{-1} \in \mathcal{A}_{t} \subseteq \underline{U}_{k}(\mathcal{A} ; t)$. If $t>\frac{1-k}{2}$, then $\mathcal{A}\left(x^{-1}\right)+t \geq \frac{1-k}{2}+\frac{1-k}{2}=1-k$, which implies that $\left[x^{-1} ; t\right] \underline{\mathrm{q}}_{k} \mathcal{A}$, i.e., $x^{-1} \in \underline{Q}_{k}(\mathcal{A} ; t) \subseteq \underline{U}_{k}(\mathcal{A} ; t)$. Now, let $x, y \in \underline{U}_{k}(\mathcal{A} ; t)$. Then we can consider the following four cases:

(i) $x, y \in \mathcal{A}_{t}$, i.e., $\mathcal{A}(x) \geq t$ and $\mathcal{A}(y) \geq t$,

(ii) $x, y \in \underline{Q}_{k}(\mathcal{A}$; $t)$, i.e., $\mathcal{A}(x)+t \geq 1-k$ and $\mathcal{A}(y)+t \geq 1-k$,

(iii) $x \in \mathcal{A}_{t}$ and $y \in \underline{Q}_{k}(\mathcal{A} ; t)$, i.e., $\mathcal{A}(x) \geq t$ and $\mathcal{A}(y)+t \geq 1-k$,

(iv) $x \in \underline{Q}_{k}(\mathcal{A} ; t)$ and $y \in \mathcal{A}_{t}$, i.e., $\mathcal{A}(x)+t \geq 1-k$ and $\mathcal{A}(y) \geq t$.

Since $\mathcal{A}$ is an $\left(\in, \in \vee \mathrm{q}_{k}\right)$-fuzzy subgroup of $G$, we have

$$
\mathcal{A}(x y) \geq \min \left\{\mathcal{A}(x), \mathcal{A}(y), \frac{1-k}{2}\right\}
$$

by Theorem 4.5(1). Using (i) and (4.2), we get $\mathcal{A}(x y) \geq \min \left\{t, \frac{1-k}{2}\right\}$. If $t \leq$ $\frac{1-k}{2}$, then $\mathcal{A}(x y) \geq t$, i.e., $x y \in \mathcal{A}_{t} \subseteq \underline{U}_{k}(\mathcal{A} ; t)$. If $t>\frac{1-k}{2}$, then $\mathcal{A}(x y) \geq \frac{1-k}{2}$ and so $\mathcal{A}(x y)+t>\frac{1-k}{2}+\frac{1-k}{2}=1-k$. Hence $x y \in Q_{k}(\mathcal{A} ; t) \subseteq \underline{Q}_{k}(\mathcal{A} ; t) \subseteq$ $\underline{U}_{k}(\mathcal{A} ; t)$. Case (ii) and (4.2) imply that $\mathcal{A}(x y) \geq \min \left\{1-k-t, \frac{1-k}{2}\right\}$. If $t \leq$ 
$\frac{1-k}{2}$, then $\mathcal{A}(x y) \geq \frac{1-k}{2} \geq t$. Hence $x y \in \mathcal{A}_{t} \subseteq \underline{U}_{k}(\mathcal{A} ; t)$. If $t>\frac{1-k}{2}$, then $\mathcal{A}(x y) \geq 1-k-t$ which implies that $x y \in \underline{Q}_{k}(\mathcal{A} ; t) \subseteq \underline{U}_{k}(\mathcal{A} ; t)$. Combining case (iii) and (4.2), we have $\mathcal{A}(x y) \geq \min \left\{t, 1-k-t, \frac{1-k}{2}\right\}$. If $t \leq \frac{1-k}{2}$, then $\mathcal{A}(x y) \geq \min \{t, 1-k-t\}=t$ and so $x y \in \mathcal{A}_{t} \subseteq \underline{U}_{k}(\mathcal{A} ; t)$. If $t>\frac{1-k}{2}$, then $\mathcal{A}(x y) \geq \min \left\{1-k-t, \frac{1-k}{2}\right\}=1-k-t$ and thus $x y \in \underline{Q}_{k}(\mathcal{A} ; t) \subseteq \underline{U}_{k}(\mathcal{A} ; t)$. Similarly we have $x y \in \underline{U}_{k}(\mathcal{A} ; t)$ from the case (iv) and $(4.2)$. Consequently, $\underline{U}_{k}(\mathcal{A} ; t)$ is a subgroup of $G$.

Conversely, suppose that (2) is valid. If there exists $x \in G$ such that $\mathcal{A}\left(x^{-1}\right)<\min \left\{\mathcal{A}(x), \frac{1-k}{2}\right\}$, then $\mathcal{A}\left(x^{-1}\right)<t \leq \min \left\{\mathcal{A}(x), \frac{1-k}{2}\right\}$ for some $t \in\left(0, \frac{1-k}{2}\right]$. It follows that $x \in \mathcal{A}_{t} \subseteq \underline{U}_{k}(\mathcal{A} ; t)$ but $x^{-1} \notin \mathcal{A}_{t}$. Also, we have $\mathcal{A}\left(x^{-1}\right)+t<2 t \leq 1-k$, and so $\left[x^{-1} ; t\right] \underline{\underline{q}}_{k} \mathcal{A}$, i.e., $x^{-1} \notin \underline{Q}_{k}(\mathcal{A} ; t)$. Therefore $x^{-1} \notin \underline{U}_{k}(\mathcal{A} ; t)$, a contradiction. Hence $\mathcal{A}\left(x^{-1}\right) \geq \min \left\{\mathcal{A}(x), \frac{1-k}{2}\right\}$ for all $x \in$ $G$. Assume that there exist $a, b \in G$ such that $\mathcal{A}(a b)<\min \left\{\mathcal{A}(a), \mathcal{A}(b), \frac{1-k}{2}\right\}$. Then $\mathcal{A}(a b)<t_{0} \leq \min \left\{\mathcal{A}(a), \mathcal{A}(b), \frac{1-k}{2}\right\}$ for some $t_{0} \in\left(0, \frac{1-k}{2}\right]$. It follows that $a, b \in \mathcal{A}_{t_{0}} \subseteq \underline{U}_{k}\left(\mathcal{A} ; t_{0}\right)$ so that $a b \in \underline{U}_{k}\left(\mathcal{A} ; t_{0}\right)$ because $\underline{U}_{k}\left(\mathcal{A} ; t_{0}\right)$ is a subgroup of $G$. Thus $\mathcal{A}(a b) \geq t_{0}$ or $\mathcal{A}(a b)+t_{0} \geq 1-k$, a contradiction. Therefore $\mathcal{A}(x y) \geq \min \left\{\mathcal{A}(x), \mathcal{A}(y), \frac{1-k}{2}\right\}$ for all $x, y \in G$. Using Theorem 4.5, we conclude that $\mathcal{A}$ is an $\left(\in, \in \vee \mathrm{q}_{k}\right)$-fuzzy subgroup of $G$.

If we take $k=0$ in Theorem 4.14, then we have the following corollary.

Corollary 4.15. A fuzzy subset $\mathcal{A}$ of $G$ is an $(\in, \in \vee \mathrm{q})$-fuzzy subgroup of $G$ if and only if it satisfies:

$(\forall t \in(0,1])(\underline{U}(\mathcal{A} ; t) \neq \emptyset \Rightarrow \underline{U}(\mathcal{A} ; t)$ is a subgroup of $G)$.

Theorem 4.16. Let $\mathcal{A}$ be an $\left(\in, \in \vee \mathrm{q}_{k}\right)$-fuzzy subgroup of $G$.

(1) If there exists $x \in G$ such that $\mathcal{A}(x) \geq \frac{1-k}{2}$, then $\mathcal{A}(e) \geq \frac{1-k}{2}$.

(2) If $\mathcal{A}(e)<\frac{1-k}{2}$, then $\mathcal{A}$ is an $(\in, \in)$-fuzzy subgroup of $G$.

Proof. (1) Suppose that $\mathcal{A}(x) \geq \frac{1-k}{2}$ for some $x \in G$. Then

$$
\mathcal{A}\left(x^{-1}\right) \geq \min \left\{\mathcal{A}(x), \frac{1-k}{2}\right\}=\frac{1-k}{2}
$$

by Theorem $4.5(2)$, and so

$$
\mathcal{A}(e)=\mathcal{A}\left(x x^{-1}\right) \geq \min \left\{\mathcal{A}(x), \mathcal{A}\left(x^{-1}\right), \frac{1-k}{2}\right\}=\frac{1-k}{2}
$$

by Theorem $4.5(1)$.

(2) Assume that $\mathcal{A}(e)<\frac{1-k}{2}$. Then $\mathcal{A}(x)<\frac{1-k}{2}$ for all $x \in G$ by (1). It follows from Theorem 4.5 that

$$
\mathcal{A}(x y) \geq \min \left\{\mathcal{A}(x), \mathcal{A}(y), \frac{1-k}{2}\right\}=\min \{\mathcal{A}(x), \mathcal{A}(y)\}
$$

and $\mathcal{A}\left(x^{-1}\right) \geq \min \left\{\mathcal{A}(x), \frac{1-k}{2}\right\}=\mathcal{A}(x)$ for all $x, y \in G$. Therefore $\mathcal{A}$ is an $(\in$, $\in)$-fuzzy subgroup of $G$.

Corollary 4.17 ([4, Theorem 3.5]). Let $\mathcal{A}$ be an $(\in, \in \vee \mathrm{q})$-fuzzy subgroup of $G$. 
(1) If there exists $x \in G$ such that $\mathcal{A}(x) \geq 0.5$, then $\mathcal{A}(e) \geq 0.5$.

(2) If $\mathcal{A}(e)<0.5$, then $\mathcal{A}$ is an $(\in, \in)$-fuzzy subgroup of $G$.

Theorem 4.18. Let $G$ be a group of prime order. If $\mathcal{A}$ is an $\left(\in, \in \vee \mathrm{q}_{k}\right)$ fuzzy subgroup of $G$ such that $\mathcal{A}(a) \geq \frac{1-k}{2}$ for some element $a(\neq e) \in G$, then $\mathcal{A}(x) \geq \frac{1-k}{2}$ for all $x \in G$.

Proof. Let $x \in G$ and assume that there exists an element $a(\neq e) \in G$ such that $\mathcal{A}(a) \geq \frac{1-k}{2}$. Then $G=(a)$, and so $x=a^{m}$ for some positive integer $m$. Using Theorem 4.5 , we have

$$
\begin{aligned}
& \mathcal{A}\left(a^{2}\right) \geq \min \left\{\mathcal{A}(a), \mathcal{A}(a), \frac{1-k}{2}\right\}=\frac{1-k}{2}, \\
& \mathcal{A}\left(a^{3}\right) \geq \min \left\{\mathcal{A}\left(a^{2}\right), \mathcal{A}(a), \frac{1-k}{2}\right\}=\frac{1-k}{2},
\end{aligned}
$$

and so on. Generally, we get $\mathcal{A}\left(a^{n}\right) \geq \frac{1-k}{2}$ for every positive integer $n$, and thus $\mathcal{A}(x) \geq \frac{1-k}{2}$ for all $x \in G$.

Corollary 4.19 ([4, Theorem 3.6]). Let $G$ be a group of prime order and let $\mathcal{A}$ be an $(\in, \in \vee \mathrm{q})$-fuzzy subgroup of $G$. If there exists one element $a(\neq e) \in G$ such that $\mathcal{A}(a) \geq 0.5$, then $\mathcal{A}(x) \geq 0.5$ for all $x \in G$.

Theorem 4.20. Let $G_{0} \subseteq G_{1} \subseteq \cdots \subseteq G_{r}=G$ be a chain of subgroups of $G$. Then there exists an $\left(\in, \in \vee \mathrm{q}_{k}\right)$-fuzzy subgroup of $G$ whose $\left(\in \vee \mathrm{q}_{k}\right)$-level subgroups are precisely the members of the chain.

Proof. Let $\left\{t_{i} \in\left(0, \frac{1-k}{2}\right] \mid i=1,2, \ldots, r\right\}$ be such that $t_{1}>t_{2}>\cdots>t_{r}$. Define a fuzzy subset $\mathcal{A}$ of $G$ by

Then

$$
\mathcal{A}: G \rightarrow[0,1], x \mapsto \begin{cases}t_{e}\left(>\frac{1-k}{2}\right) & \text { if } x=e, \\ t\left(>t_{e}\right)^{2} & \text { if } x \in G_{0} \backslash\{e\} \\ t_{1} & \text { if } x \in G_{1} \backslash G_{0}, \\ t_{2} & \text { if } x \in G_{2} \backslash G_{1}, \\ \cdots & \text { if } x \in G_{r} \backslash G_{r-1} . \\ t_{r} & \end{cases}
$$

$$
\mathcal{A}_{s}= \begin{cases}G_{0} & \text { if } s \in\left(t_{1}, \frac{1-k}{2}\right] \\ G_{1} & \text { if } s \in\left(t_{2}, t_{1}\right] \\ G_{2} & \text { if } s \in\left(t_{3}, t_{2}\right] \\ \cdots & \text { if } s \in\left(0, t_{r}\right] \\ G_{r}=G & \text { is }\end{cases}
$$

Using Theorem 4.10, we know that $\mathcal{A}$ is an $\left(\in, \in \vee \mathrm{q}_{k}\right)$-fuzzy subgroup of $G$, and clearly whose $\left(\in \vee \mathrm{q}_{k}\right)$-level subgroups are precisely the members of the chain.

Combining Theorems 2.2 and 4.20, we know that if $G_{0}=\{e\}$, then $\mathcal{A}$ defined in Theorem 4.20 is an $(\epsilon, \in)$-fuzzy subgroup of $G$.

If we take $k=0$ in Theorem 4.20 , then we have the following corollary. 
Corollary 4.21 ([4]). Let $G_{0} \subseteq G_{1} \subseteq \cdots \subseteq G_{r}=G$ be a chain of subgroups of $G$. Then there exists an $(\in, \in \vee \mathrm{q})$-fuzzy subgroup of $G$ whose $(\in \vee \mathrm{q})$-level subgroups are precisely the members of the chain.

Proposition 4.22. Let $\mathcal{A}$ be an $\left(\in, \in \vee \mathrm{q}_{k}\right)$-fuzzy subgroup of $G$ and let $x, y \in$ $G$ such that $\mathcal{A}(x)<\mathcal{A}(y)$. Then

(1) $\mathcal{A}(x) \geq \frac{1-k}{2} \Rightarrow \min \{\mathcal{A}(x y), \mathcal{A}(y x)\} \geq \frac{1-k}{2}$.

(2) $\mathcal{A}(x)<\frac{1-k}{2} \Rightarrow \mathcal{A}(x y)=\mathcal{A}(x)=\mathcal{A}(y x)$.

Proof. (1) If $\mathcal{A}(x) \geq \frac{1-k}{2}$, then $\mathcal{A}(y) \geq \frac{1-k}{2}$, and so $x, y \in \mathcal{A}_{\frac{1-k}{2}}$. Since $\mathcal{A}_{\frac{1-k}{2}}$ is a subgroup of $G$ by Theorem 4.10, it follows that $x y, y x \in \frac{1}{\epsilon} \mathcal{A}_{\frac{1-k}{2}}$ so that $\min \{\mathcal{A}(x y), \mathcal{A}(y x)\} \geq \frac{1-k}{2}$.

(2) Suppose that $\mathcal{A}(x)<\frac{1-k}{2}$. Then

$$
\mathcal{A}(x y) \geq \min \left\{\mathcal{A}(x), \mathcal{A}(y), \frac{1-k}{2}\right\}=\mathcal{A}(x)
$$

by Theorem 4.5(1) and hypothesis. Now, we have

$$
\mathcal{A}(x)=\mathcal{A}\left(x y y^{-1}\right) \geq \min \left\{\mathcal{A}(x y), \mathcal{A}\left(y^{-1}\right), \frac{1-k}{2}\right\} \geq \min \left\{\mathcal{A}(x y), \mathcal{A}(y), \frac{1-k}{2}\right\}
$$

by Theorem 4.5. Since $\mathcal{A}(x)<\mathcal{A}(y)$ and $\mathcal{A}(x)<\frac{1-k}{2}$, it follows that $\mathcal{A}(x) \geq$ $\mathcal{A}(x y)$. Hence $\mathcal{A}(x y)=\mathcal{A}(x)$. Similarly, one can show that $\mathcal{A}(y x)=\mathcal{A}(x)$. This completes the proof.

Theorem 4.23. Let $\mathcal{A}$ be an $\left(\in, \in \vee \mathrm{q}_{k}\right)$-fuzzy subgroup of $G$ with $\operatorname{Im}(\mathcal{A})=$ $\left\{t_{1}, t_{2}\right\}$ where $t_{i} \in\left(0, \frac{1-k}{2}\right)$ for $i=1,2$. If $\mathcal{A}$ can be realized as a union of two $\left(\in, \in \vee \mathrm{q}_{k}\right)$-fuzzy subgroups $\mathcal{B}$ and $\mathcal{C}$ of $G$, then either $\mathcal{B} \subseteq \mathcal{C}$ or $\mathcal{C} \subseteq \mathcal{B}$.

Proof. Since $\mathcal{A}(e)<\frac{1-k}{2}$ where $e$ is the identity element of $G$, it follows from Theorem $4.16(2)$ that $\mathcal{A}$ is an $(\in, \in)$-fuzzy subgroup of $G$. Thus the result follows from [5, Proposition 3.2].

If $t_{1}<\frac{1-k}{2}<t_{2}$, then $\mathcal{A}$ can be expressed as the union of two $\left(\in, \in \vee \mathrm{q}_{k}\right)$ fuzzy subgroups $\mathcal{B}$ and $\mathcal{C}$ of $G$ with $\mathcal{B} \nsubseteq \mathcal{C}$ and $\mathcal{C} \nsubseteq \mathcal{B}$.

Example 4.24. In Example 4.9, let $\mathcal{A}, \mathcal{B}$ and $\mathcal{C}$ be fuzzy subsets of $G$ defined by

$$
\begin{aligned}
\mathcal{A} & =\left(\begin{array}{cccc}
e & a & b & c \\
0.6 & 0.6 & 0.6 & 0.2
\end{array}\right), \\
\mathcal{B} & =\left(\begin{array}{cccc}
e & a & b & c \\
0.6 & 0.6 & 0.2 & 0.2
\end{array}\right), \\
\mathcal{C} & =\left(\begin{array}{cccc}
e & a & b & c \\
0.6 & 0.2 & 0.6 & 0.2
\end{array}\right),
\end{aligned}
$$

respectively. Then $\mathcal{A}, \mathcal{B}$ and $\mathcal{C}$ are $\left(\in, \in \vee \mathrm{q}_{k}\right)$-fuzzy subgroups of $G$ for $k=0.2$ and $\mathcal{A}=\mathcal{B} \cup \mathcal{C}$ but $\mathcal{B} \nsubseteq \mathcal{C}$ and $\mathcal{C} \nsubseteq \mathcal{B}$.

Let $H$ be a subset of $G$. The subgroup generated by $H$ in $G$ is denoted by $\langle H\rangle$. 
Definition 4.25. Let $\mathcal{A}$ be a fuzzy subset of $G$. An $\left(\in, \in \vee \mathrm{q}_{k}\right)$-fuzzy subgroup $\mathcal{B}$ of $G$ is said to be the $\left(\in, \in \vee \mathrm{q}_{k}\right)$-fuzzy subgroup generated by $\mathcal{A}$ in $G$ if $\mathcal{A} \subseteq \mathcal{B}$ and for any $\left(\in, \in \vee \mathrm{q}_{k}\right)$-fuzzy subgroup $\mathcal{C}$ of $G$ with $\mathcal{A} \subseteq \mathcal{C}$ it must be $\mathcal{B} \subseteq \mathcal{C}$.

Theorem 4.26. Let $\mathcal{A}$ be a fuzzy subset of $G$ with $\operatorname{Card} \operatorname{Im}(\mathcal{A})<\infty$. Define subgroups $G_{i}$ of $G$ as follows:

$$
\begin{aligned}
& G_{0}=\left\langle\left\{x \in G \mid \mathcal{A}(x) \geq \frac{1-k}{2}\right\}\right\rangle, \\
& G_{i}=\left\langleG _ { i - 1 } \cup \left\{ x \in G\left|\mathcal{A}(x)=\sup \left\{\mathcal{A}(z) \mid z \in G \backslash G_{i-1}\right\}\right\rangle\right.\right.
\end{aligned}
$$

for $i=1,2, \ldots, k$ where $k<\operatorname{Card} \operatorname{Im}(\mathcal{A})$ and $G_{k}=G$. Let $\mathcal{A}^{*}$ be a fuzzy subset of $G$ defined by

$$
\mathcal{A}^{*}(x):= \begin{cases}\mathcal{A}(x) & \text { if } x \in G_{0}, \\ \sup \left\{\mathcal{A}(z) \mid z \in G \backslash G_{i-1}\right\} & \text { if } x \in G_{i} \backslash G_{i-1} .\end{cases}
$$

Then $\mathcal{A}^{*}$ is the $\left(\in, \in \vee \mathrm{q}_{k}\right)$-fuzzy subgroup generated by $\mathcal{A}$ in $G$.

Proof. Obviously, $\mathcal{A} \subseteq \mathcal{A}^{*}$ by the construction of $\mathcal{A}^{*}$. Furthermore, the $G_{i}$ 's from a chain

$$
G_{0} \subseteq G_{1} \subseteq \cdots \subseteq G_{k}=G
$$

of subgroups ending at $G$. We first show that $\mathcal{A}^{*}$ is an $\left(\in, \in \vee \mathrm{q}_{k}\right)$-fuzzy subgroup of $G$. Let $x, y \in G$. If $x, y \in G_{0}$, then $x y \in G_{0}$ and $x^{-1} \in G_{0}$ since $G_{0}$ is a subgroup of $G$. Using Theorem 4.5 , we have

$$
\mathcal{A}^{*}(x y)=\mathcal{A}(x y) \geq \min \left\{\mathcal{A}(x), \mathcal{A}(y), \frac{1-k}{2}\right\}=\min \left\{\mathcal{A}^{*}(x), \mathcal{A}^{*}(y), \frac{1-k}{2}\right\}
$$

and

$$
\mathcal{A}^{*}\left(x^{-1}\right)=\mathcal{A}\left(x^{-1}\right) \geq \min \left\{\mathcal{A}(x), \frac{1-k}{2}\right\}=\min \left\{\mathcal{A}^{*}(x), \frac{1-k}{2}\right\} .
$$

Now let $x \in G_{i} \backslash G_{i-1}$ and $y \in G_{j} \backslash G_{j-1}$. We may assume that $i<j$ without loss of generality. Then $x, y \in G_{j}$ and so $x y, x^{-1} \in G_{j}$. It follows that

$$
\begin{aligned}
\mathcal{A}^{*}(x y) & \geq \sup \left\{\mathcal{A}(z) \mid z \in G \backslash G_{j-1}\right\} \\
& \geq \min \left\{\sup \left\{\mathcal{A}(z) \mid z \in G \backslash G_{i-1}\right\}, \sup \left\{\mathcal{A}(z) \mid z \in G \backslash G_{j-1}\right\}, \frac{1-k}{2}\right\} \\
& =\min \left\{\mathcal{A}^{*}(x), \mathcal{A}^{*}(y), \frac{1-k}{2}\right\}
\end{aligned}
$$

and

$$
\begin{aligned}
\mathcal{A}^{*}\left(x^{-1}\right) & \geq \sup \left\{\mathcal{A}(z) \mid z \in G \backslash G_{j-1}\right\} \\
& \geq \min \left\{\sup \left\{\mathcal{A}(z) \mid z \in G \backslash G_{i-1}\right\}, \frac{1-k}{2}\right\} \\
& =\min \left\{\mathcal{A}^{*}(x), \frac{1-k}{2}\right\} .
\end{aligned}
$$

Hence $\mathcal{A}^{*}$ is an $\left(\in, \in \vee \mathrm{q}_{k}\right)$-fuzzy subgroup of $G$ whose $\left(\in \vee \mathrm{q}_{k}\right)$-level subgroups are precisely the members of the above chain by Theorem 4.20. Now, let $\mathcal{B}$ be any $\left(\in, \in \vee q_{k}\right)$-fuzzy subgroup of $G$ with $\mathcal{A} \subseteq \mathcal{B}$. If $x \in G_{0}$, then $\mathcal{A}^{*}(x)=\mathcal{A}(x) \leq \mathcal{B}(x)$. Let $\left\{B_{t_{i}}\right\}$ be the class of $\left(\in \vee \mathrm{q}_{k}\right)$-level subgroups of $\mathcal{B}$ in $G$. Let $x \in G_{1} \backslash G_{0}$. Then $\mathcal{A}^{*}(x)=\sup \left\{\mathcal{A}(z) \mid z \in G \backslash G_{0}\right\}$ and $G_{1}=\left\langle K_{1}\right\rangle$ where

$$
K_{1}=G_{0} \cup\left\{x \in G \mid \mathcal{A}(x)=\sup \left\{\mathcal{A}(z) \mid z \in G \backslash G_{0}\right\}\right\}
$$


TABLE 3. Multiplication table for $S_{3}$

\begin{tabular}{l|llllll}
\hline & 1 & $(123)$ & $(132)$ & $(23)$ & $(13)$ & $(12)$ \\
\hline 1 & 1 & $(123)$ & $(132)$ & $(23)$ & $(13)$ & $(12)$ \\
$(123)$ & $(123)$ & $(132)$ & 1 & $(13)$ & $(12)$ & $(23)$ \\
$(132)$ & $(132)$ & 1 & $(123)$ & $(12)$ & $(23)$ & $(13)$ \\
$(23)$ & $(23)$ & $(12)$ & $(13)$ & 1 & $(132)$ & $(123)$ \\
$(13)$ & $(13)$ & $(23)$ & $(12)$ & $(123)$ & 1 & $(132)$ \\
$(12)$ & $(12)$ & $(13)$ & $(23)$ & $(132)$ & $(123)$ & 1 \\
\hline
\end{tabular}

Let $x \in K_{1} \backslash G_{0}$. Then $\left.\mathcal{A}(x)=\sup \left\{\mathcal{A}(z) \mid z \in G \backslash G_{0}\right\}\right\}$. Since $\mathcal{A} \subseteq \mathcal{B}$, it follows that

$$
\sup \left\{\mathcal{A}(z) \mid z \in G \backslash G_{0}\right\} \leq \inf \left\{\mathcal{B}(x) \mid x \in K_{1} \backslash G_{0}\right\} \leq \mathcal{B}(x) .
$$

Putting $t_{i 1}=\inf \left\{\mathcal{B}(x) \mid x \in K_{1} \backslash G_{0}\right\}$, we have $x \in \mathcal{B}_{t_{i 1}}$ and hence $K_{1} \backslash G_{0} \subseteq$ $\mathcal{B}_{t_{i 1}}$. Since $G_{0} \subseteq \mathcal{B}_{t_{i 1}}$, we get $G_{1}=\left\langle K_{1}\right\rangle \subseteq \mathcal{B}_{t_{i 1}}$. Thus $\mathcal{B}(x) \geq t_{i 1}$ for all $x \in G_{1}$. Therefore

$$
\mathcal{A}^{*}(x)=\sup \left\{\mathcal{A}(z) \mid z \in G \backslash G_{0}\right\} \leq t_{i 1} \leq \mathcal{B}(x)
$$

for all $x \in G_{1} \backslash G_{0}$. Similarly, we can prove that $\mathcal{A}^{*}(x) \leq \mathcal{B}(x)$ for all $x \in$ $G_{i} \backslash G_{i-1}$ where $2 \leq i \leq k$. Consequently, $\mathcal{A}^{*}$ is the $\left(\in, \in \vee \mathrm{q}_{k}\right)$-fuzzy subgroup generated by $\mathcal{A}$ in $G$.

\section{5. $\left(\in, \in \vee \mathrm{q}_{k}\right)$-fuzzy normal subgroups}

Definition 5.1 ([2]). A fuzzy subset $\mathcal{A}$ of $G$ is called an $(\in, \in)$-fuzzy normal subgroup of $G$ if it is an $(\in, \in)$-fuzzy subgroup of $G$ that satisfies:

$$
(\forall x, y \in G)(\forall t \in(0,1])\left([x ; t] \in \mathcal{A} \Rightarrow\left[y^{-1} x y ; t\right] \in \mathcal{A}\right) .
$$

Definition $5.2([2])$. A fuzzy subset $\mathcal{A}$ of $G$ is called an $(\in, \in \vee \mathrm{q})$-fuzzy normal subgroup of $G$ if it is an $(\in, \in \vee \mathrm{q})$-fuzzy subgroup of $G$ that satisfies:

$$
(\forall x, y \in G)(\forall t \in(0,1])\left([x ; t] \in \mathcal{A} \Rightarrow\left[y^{-1} x y ; t\right] \in \vee \mathrm{q} \mathcal{A}\right) .
$$

Definition 5.3. A fuzzy subset $\mathcal{A}$ of $G$ is called an $\left(\in, \in \vee \mathrm{q}_{k}\right)$-fuzzy normal subgroup of $G$ if it is an $\left(\in, \in \vee \mathrm{q}_{k}\right)$-fuzzy subgroup of $G$ that satisfies:

$$
(\forall x, y \in G)(\forall t \in(0,1])\left([x ; t] \in \mathcal{A} \Rightarrow\left[y^{-1} x y ; t\right] \in \vee \mathrm{q}_{k} \mathcal{A}\right) .
$$

An $\left(\in, \in \vee \mathrm{q}_{k}\right)$-fuzzy normal subgroup of $G$ with $k=0$ is called an $(\in$, $\in \vee \mathrm{q}$ )-fuzzy normal subgroup of $G$. Every $(\epsilon, \in)$-fuzzy normal subgroup is an $\left(\in, \in \vee \mathrm{q}_{k}\right)$-fuzzy normal subgroup. However, the converse may not be true.

Example 5.4. Consider the symmetric group

$$
S_{3}:=\{1,(123),(132),(23),(13),(12)\}
$$

of degree 3 in which the multiplication is defined by Table 3 . Let $\mathcal{A}$ be a fuzzy 
subset of $S_{3}$ defined by

$$
\mathcal{A}=\left(\begin{array}{cccccc}
1 & (123) & (132) & (23) & (13) & (12) \\
0.4 & 0.5 & 0.8 & 0.1 & 0.1 & 0.1
\end{array}\right)
$$

Then $\mathcal{A}$ is an $\left(\in, \in \vee \mathrm{q}_{k}\right)$-fuzzy normal subgroup of $S_{3}$ for $k=0.4$. But, since $\mathcal{A}((12)(13))=\mathcal{A}((123))=0.5 \neq 0.8=\mathcal{A}((132))=\mathcal{A}((13)(12)), \mathcal{A}$ is not an $(\in, \in)$-fuzzy normal subgroup of $S_{3}$. Let

$$
\Omega:=\left\{k \in[0,1) \mid \mathcal{A} \text { is an }\left(\in, \in \vee \mathrm{q}_{k}\right) \text {-fuzzy normal subgroup of } S_{3}\right\} .
$$

Then $\inf \Omega$ is equal to 0.2 .

Theorem 5.5. For an $\left(\in, \in \vee q_{k}\right)$-fuzzy subgroup $\mathcal{A}$ of $G$, the following are equivalent.

(1) $\mathcal{A}$ is $\left(\in, \in \vee \mathrm{q}_{k}\right)$-fuzzy normal.

(2) $(\forall x, y \in G)\left(\mathcal{A}\left(y^{-1} x y\right) \geq \min \left\{\mathcal{A}(x), \frac{1-k}{2}\right\}\right)$.

(3) $(\forall x, y \in G)\left(\mathcal{A}(x y) \geq \min \left\{\mathcal{A}(y x), \frac{1-k}{2}\right\}\right)$.

(4) $(\forall x, y \in G)\left(\mathcal{A}\left(x^{-1} y^{-1} x y\right) \geq \min \left\{\mathcal{A}(x), \frac{1-k}{2}\right\}\right)$.

Proof. (1) $\Rightarrow(2)$. Suppose that there exist $a, b \in G$ such that $\mathcal{A}\left(b^{-1} a b\right)<$ $\min \left\{\mathcal{A}(a), \frac{1-k}{2}\right\}$. Then $\mathcal{A}\left(b^{-1} a b\right)<t \leq \min \left\{\mathcal{A}(a), \frac{1-k}{2}\right\}$ for some $t \in\left(0, \frac{1-k}{2}\right]$. It follows that $[a ; t] \in \mathcal{A}$ and $\left[b^{-1} a b ; t\right] \bar{\in} \mathcal{A}$. Moreover, $\mathcal{A}\left(b^{-1} a b\right)+t<2 t \leq$ $1-k$, and so $\left[b^{-1} a b ; t\right] \overline{\mathrm{q}_{k}} \mathcal{A}$. Therefore $\left[b^{-1} a b ; t\right] \overline{\in \vee \mathrm{q}_{k}} \mathcal{A}$, a contradiction. Hence $\mathcal{A}\left(y^{-1} x y\right) \geq \min \left\{\mathcal{A}(x), \frac{1-k}{2}\right\}$ for all $x, y \in G$.

$(2) \Rightarrow(1)$. Let $x, y \in G$ and $t \in(0,1]$ be such that $[x ; t] \in \mathcal{A}$. Then $\mathcal{A}(x) \geq t$, and thus

$$
\mathcal{A}\left(y^{-1} x y\right) \geq \min \left\{\mathcal{A}(x), \frac{1-k}{2}\right\} \geq \min \left\{t, \frac{1-k}{2}\right\} .
$$

Suppose that $\left[y^{-1} x y ; t\right] \bar{\in} \mathcal{A}$, i.e., $\mathcal{A}\left(y^{-1} x y\right)<t$. If $t<\frac{1-k}{2}$, then $\mathcal{A}\left(y^{-1} x y\right) \geq t$ by (5.1). This is a contradiction, and so $t \geq \frac{1-k}{2}$. Therefore

$$
\mathcal{A}\left(y^{-1} x y\right)+t>2 \mathcal{A}\left(y^{-1} x y\right) \geq 2 \min \left\{t, \frac{1-k}{2}\right\}=1-k,
$$

i.e., $\left[y^{-1} x y ; t\right] \mathrm{q}_{k} \mathcal{A}$. Hence $\left[y^{-1} x y ; t\right] \in \vee \mathrm{q}_{k} \mathcal{A}$ and consequently $\mathcal{A}$ is $(\in, \in$ $\vee \mathrm{q}_{k}$ )-fuzzy normal.

$(2) \Leftrightarrow(3)$. Using (2), we have $\mathcal{A}(x y)=\mathcal{A}\left(y^{-1} y x y\right) \geq \min \left\{\mathcal{A}(y x), \frac{1-k}{2}\right\}$ for all $x, y \in G$. Now (3) implies that

$$
\mathcal{A}\left(y^{-1} x y\right) \geq \min \left\{\mathcal{A}\left(y y^{-1} x\right), \frac{1-k}{2}\right\}=\min \left\{\mathcal{A}(x), \frac{1-k}{2}\right\}
$$

for all $x, y \in G$.

$(3) \Rightarrow(4)$. Using Theorem 4.5 and $(3)$, we get

$$
\begin{aligned}
\mathcal{A}\left(x^{-1} y^{-1} x y\right) & \geq \min \left\{\mathcal{A}\left(x^{-1}\right), \mathcal{A}\left(y^{-1} x y\right), \frac{1-k}{2}\right\} \\
& \geq \min \left\{\min \left\{\mathcal{A}(x), \frac{1-k}{2}\right\}, \min \left\{\mathcal{A}\left(x y y^{-1}\right), \frac{1-k}{2}\right\}, \frac{1-k}{2}\right\} \\
& =\min \left\{\mathcal{A}(x), \frac{1-k}{2}\right\}
\end{aligned}
$$

for all $x, y \in G$. 
$(4) \Rightarrow(2)$. Using Theorem 4.5 and (4), we obtain

$$
\begin{aligned}
\mathcal{A}\left(y^{-1} x y\right) & =\mathcal{A}\left(x x^{-1} y^{-1} x y\right) \geq \min \left\{\mathcal{A}(x), \mathcal{A}\left(x^{-1} y^{-1} x y\right), \frac{1-k}{2}\right\} \\
& \geq \min \left\{\mathcal{A}(x), \min \left\{\mathcal{A}(x), \frac{1-k}{2}\right\}, \frac{1-k}{2}\right\} \\
& =\min \left\{\mathcal{A}(x), \frac{1-k}{2}\right\}
\end{aligned}
$$

for all $x, y \in G$. This completes the proof.

Corollary 5.6 ([4]). For an $(\in, \in \vee \mathrm{q})$-fuzzy subgroup $\mathcal{A}$ of $G$, the following assertions are equivalent.

(1) $\mathcal{A}$ is $(\in, \in \vee \mathrm{q})$-fuzzy normal.

(2) $(\forall x, y \in G)\left(\mathcal{A}\left(y^{-1} x y\right) \geq \min \{\mathcal{A}(x), 0.5\}\right)$.

(3) $(\forall x, y \in G)(\mathcal{A}(x y) \geq \min \{\mathcal{A}(y x), 0.5\})$.

(4) $(\forall x, y \in G)\left(\mathcal{A}\left(x^{-1} y^{-1} x y\right) \geq \min \{\mathcal{A}(x), 0.5\}\right)$.

Theorem 5.7. For any fuzzy subset $\mathcal{A}$ of $G$, the following are equivalent:

(1) $\mathcal{A}$ is an $\left(\in, \in \vee \mathrm{q}_{k}\right)$-fuzzy normal subgroup of $G$.

(2) $\left(\forall t \in\left(0, \frac{1-k}{2}\right]\right)\left(\mathcal{A}_{t} \neq \emptyset \Rightarrow \mathcal{A}_{t}\right.$ is a normal subgroup of $\left.G\right)$.

Proof. Let $\mathcal{A}$ be an $\left(\in, \in \vee \mathrm{q}_{k}\right)$-fuzzy normal subgroup of $G$ and let $t \in\left(0, \frac{1-k}{2}\right]$ such that $\mathcal{A}_{t} \neq \emptyset$. Then $\mathcal{A}_{t}$ is a subgroup of $G$ by Theorem 4.10 . We now show that $\mathcal{A}_{t}$ is normal. Let $x \in \mathcal{A}_{t}$ and $y \in G$. Then $\mathcal{A}(x) \geq t$ and so

$$
\mathcal{A}\left(y^{-1} x y\right) \geq \min \left\{\mathcal{A}(x), \frac{1-k}{2}\right\} \geq \min \left\{t, \frac{1-k}{2}\right\}=t
$$

by Theorem 5.5. Hence $y^{-1} x y \in \mathcal{A}_{t}$, that is, $\mathcal{A}_{t}$ is normal.

Conversely, let $\mathcal{A}$ be a fuzzy subset of $G$ such that the nonempty level set $\mathcal{A}_{t}$ is a normal subgroup of $G$ for all $t \in\left(0, \frac{1-k}{2}\right]$. Using Theorem $4.10, \mathcal{A}$ is an $\left(\in, \in \vee \mathrm{q}_{k}\right)$-fuzzy subgroup of $G$. Assume that $\mathcal{A}\left(b^{-1} a b\right)<\min \left\{\mathcal{A}(a), \frac{1-k}{2}\right\}$ for some $a, b \in G$. Then there exists $t \in\left(0, \frac{1-k}{2}\right]$ such that $\mathcal{A}\left(b^{-1} a b\right)<$ $t \leq \min \left\{\mathcal{A}(a), \frac{1-k}{2}\right\}$. Then $a \in \mathcal{A}_{t}$ but $b^{-1} a b \notin \mathcal{A}_{t}$, a contradiction. Thus $\mathcal{A}\left(y^{-1} x y\right) \geq \min \left\{\mathcal{A}(x), \frac{1-k}{2}\right\}$ for all $x, y \in G$. It follows from Theorem 5.5 that $\mathcal{A}$ is an $\left(\in, \in \vee \mathrm{q}_{k}\right)$-fuzzy normal subgroup of $G$.

If we take $k=0$ in Theorem 5.7, then we have the following corollary.

Corollary 5.8 ([4]). Let $\mathcal{A}$ be an $(\in, \in \vee \mathrm{q})$-fuzzy normal subgroup of $G$. Then $\mathcal{A}_{t}$ is a normal subgroup of $G$ for all $t \leq 0.5$. Conversely, if $\mathcal{A}$ is a fuzzy subset of $G$ such that $\mathcal{A}_{t}$ is a normal subgroup of $G$ for all $t \leq 0.5$, then $\mathcal{A}$ is an $(\in$, $\in \vee$ q)-fuzzy normal subgroup of $G$.

Definition 5.9. Let $\mathcal{A}$ be a fuzzy subset of $G$. For any $x \in G$, the fuzzy subset

$$
\begin{gathered}
\mathcal{A}_{x}^{l}: G \rightarrow[0,1], y \mapsto \mathcal{A}\left(y x^{-1}\right) \\
\text { (resp. } \mathcal{A}_{x}^{r}: G \rightarrow[0,1], y \mapsto \mathcal{A}\left(x^{-1} y\right) \text { ) }
\end{gathered}
$$

is called the fuzzy left (resp. right) coset of $G$ determined by $x$ and $\mathcal{A}$. 
If $\mathcal{A}$ is an $(\in, \in)$-fuzzy subgroup of $G$, then $\mathcal{A}$ is an $(\in, \in)$-fuzzy normal if and only if $\mathcal{A}_{x}^{l}=\mathcal{A}_{x}^{r}$ for all $x \in G$. However, if $\mathcal{A}$ is an $\left(\in, \in \vee \mathrm{q}_{k}\right)$-fuzzy subgroup of $G$, then $\mathcal{A}_{x}^{l}$ may not be equal to $\mathcal{A}_{x}^{r}$ as shown by the following example.

Example 5.10. Consider the $\left(\in, \in \vee q_{k}\right)$-fuzzy normal subgroup $\mathcal{A}$ of $S_{3}$ for $k=0.4$ as defined in Example 5.4. Then

$$
\mathcal{A}_{(13)}^{l}((23))=\mathcal{A}\left((23)(13)^{-1}\right)=\mathcal{A}((23)(13))=\mathcal{A}((132))=0.8
$$

and

$$
\mathcal{A}_{(13)}^{r}((23))=\mathcal{A}\left((13)^{-1}(23)\right)=\mathcal{A}((13)(23))=\mathcal{A}((123))=0.5 .
$$

Hence $\mathcal{A}_{(13)}^{l}((23)) \neq \mathcal{A}_{(13)}^{r}((23))$.

Definition 5.11. Let $\mathcal{A}$ be an $\left(\in, \in \vee \mathrm{q}_{k}\right)$-fuzzy subgroup of $G$. For any $x \in G$, $\overleftarrow{\mathcal{A}}_{x}\left(\operatorname{resp} . \overrightarrow{\mathcal{A}}_{x}\right): G \rightarrow[0,1]$ defined by

$$
\overleftarrow{\mathcal{A}}_{x}(y)=\min \left\{\mathcal{A}_{x}^{l}(y), \frac{1-k}{2}\right\} \quad\left(\operatorname{resp} . \overrightarrow{\mathcal{A}}_{x}(y)=\min \left\{\mathcal{A}_{x}^{r}(y), \frac{1-k}{2}\right\}\right)
$$

is called the $\left(\in, \in \vee \mathrm{q}_{k}\right)$-fuzzy left (resp. right) coset of $G$ determined by $x$ and $\mathcal{A}$.

Theorem 5.12. Let $\mathcal{A}$ be an $\left(\in, \in \vee \mathrm{q}_{k}\right)$-fuzzy subgroup of $G$. Then $\mathcal{A}$ is $(\in$, $\in \vee \mathrm{q}_{k}$ )-fuzzy normal if and only if $\overleftarrow{\mathcal{A}}_{x}=\overrightarrow{\mathcal{A}}_{x}$ for all $x \in G$

Proof. Let $\mathcal{A}$ be an $\left(\in, \in \vee \mathrm{q}_{k}\right)$-fuzzy normal subgroup of $G$. Let $x \in G$. For every $g \in G$, we have

$$
\begin{aligned}
\overleftarrow{\mathcal{A}}_{x}(g) & =\min \left\{\mathcal{A}_{x}^{l}(g), \frac{1-k}{2}\right\}=\min \left\{\mathcal{A}\left(g x^{-1}\right), \frac{1-k}{2}\right\} \\
& \geq \min \left\{\min \left\{\mathcal{A}\left(x^{-1} g\right), \frac{1-k}{2}\right\}, \frac{1-k}{2}\right\} \\
& =\min \left\{\mathcal{A}\left(x^{-1} g\right), \frac{1-k}{2}\right\}=\min \left\{\mathcal{A}_{x}^{r}(g), \frac{1-k}{2}\right\} \\
& =\overrightarrow{\mathcal{A}}_{x}(g)
\end{aligned}
$$

by using Theorem 5.5. Similarly, $\overleftarrow{\mathcal{A}}_{x}(g) \leq \overrightarrow{\mathcal{A}}_{x}(g)$ and so $\overleftarrow{\mathcal{A}}_{x}(g)=\overrightarrow{\mathcal{A}}_{x}(g)$ for all $x \in G$.

Conversely, assume that $\overleftarrow{\mathcal{A}}_{x}=\overrightarrow{\mathcal{A}}_{x}$ for all $x \in G$. Then

$$
\min \left\{\mathcal{A}_{x}^{l}(g), \frac{1-k}{2}\right\}=\min \left\{\mathcal{A}_{x}^{r}(g), \frac{1-k}{2}\right\},
$$

that is, $\min \left\{\mathcal{A}\left(g x^{-1}\right), \frac{1-k}{2}\right\}=\min \left\{\mathcal{A}\left(x^{-1} g\right), \frac{1-k}{2}\right\}$ for all $g \in G$. Taking $g=$ $x y x$ implies that $\mathcal{A}(x y) \geq \min \left\{\mathcal{A}(y x), \frac{1-k}{2}\right\}$. Using Theorem 5.5, we conclude that $\mathcal{A}$ is $\left(\in, \in \vee \mathrm{q}_{k}\right)$-fuzzy normal.

When $\mathcal{A}$ is an $\left(\in, \in \vee \mathrm{q}_{k}\right)$-fuzzy normal the $\left(\in, \in \vee \mathrm{q}_{k}\right)$-fuzzy coset of $G$ determined by $x$ and $\mathcal{A}$ is denoted by $\mathcal{A}_{x}$. Let $\mathcal{G}(\mathcal{A})$ be the set of all $(\in$, $\in \vee \mathrm{q}_{k}$ )-fuzzy cosets of $\mathcal{A}$ in $G$. Define a multiplication "." on $\mathcal{G}(\mathcal{A})$ by

$$
\mathcal{A}_{x} \cdot \mathcal{A}_{y}=\mathcal{A}_{x y}
$$


for all $\mathcal{A}_{x}, \mathcal{A}_{y} \in \mathcal{G}(\mathcal{A})$. Let $\mathcal{A}_{x}=\mathcal{A}_{y}$ and $\mathcal{A}_{u}=\mathcal{A}_{v}$ where $x, y, u, v \in G$. Then $\mathcal{A}_{x}(g)=\mathcal{A}_{y}(g)$ and $\mathcal{A}_{u}(g)=\mathcal{A}_{v}(g)$, i.e.,

$$
\min \left\{\mathcal{A}\left(x^{-1} g\right), \frac{1-k}{2}\right\}=\min \left\{\mathcal{A}\left(y^{-1} g\right), \frac{1-k}{2}\right\}
$$

and

$$
\min \left\{\mathcal{A}\left(u^{-1} g\right), \frac{1-k}{2}\right\}=\min \left\{\mathcal{A}\left(v^{-1} g\right), \frac{1-k}{2}\right\}
$$

for all $g \in G$. Replacing $g$ by $x^{-1} g$ (resp. $g v^{-1}$ ) in (5.4) (resp. (5.3)) and using Theorem 5.5, we have

$$
\begin{aligned}
\mathcal{A}_{x u}(g) & =\min \left\{\mathcal{A}\left((x u)^{-1} g\right), \frac{1-k}{2}\right\}=\min \left\{\mathcal{A}\left(u^{-1} x^{-1} g\right), \frac{1-k}{2}\right\} \\
& =\min \left\{\mathcal{A}\left(v^{-1} x^{-1} g\right), \frac{1-k}{2}\right\} \\
& \geq \min \left\{\min \left\{\mathcal{A}\left(x^{-1} g v^{-1}\right), \frac{1-k}{2}\right\}, \frac{1-k}{2}\right\} \\
& =\min \left\{\mathcal{A}\left(x^{-1} g v^{-1}\right), \frac{1-k}{2}\right\}=\min \left\{\mathcal{A}\left(y^{-1} g v^{-1}\right), \frac{1-k}{2}\right\} \\
& \geq \min \left\{\min \left\{\mathcal{A}\left(v^{-1} y^{-1} g\right), \frac{1-k}{2}\right\}, \frac{1-k}{2}\right\} \\
& =\min \left\{\mathcal{A}\left((y v)^{-1} g\right), \frac{1-k}{2}\right\} \\
& =\mathcal{A}_{y v}(g) .
\end{aligned}
$$

Similarly, one can show that $\mathcal{A}_{y v}(g) \geq \mathcal{A}_{x u}(g)$ for all $g \in G$. Hence $\mathcal{A}_{x u}=\mathcal{A}_{y v}$, which shows that $(5.2)$ is well defined. It can be easily verified that $\mathcal{G}(\mathcal{A})$ is a group with $\mathcal{A}_{e}$ as the identity element and $\mathcal{A}_{x}^{-1}=\mathcal{A}_{x^{-1}}$ for any $\mathcal{A}_{x} \in \mathcal{G}(\mathcal{A})$.

Theorem 5.13. Let $\mathcal{A}$ be an $\left(\in, \in \vee \mathrm{q}_{k}\right)$-fuzzy normal subgroup of $G$. Then the fuzzy subset $\tilde{\mathcal{A}}$ of $\mathcal{G}(\mathcal{A})$ defined by

$$
\tilde{\mathcal{A}}: \mathcal{G}(\mathcal{A}) \rightarrow[0,1], \mathcal{A}_{x} \mapsto \mathcal{A}(x)
$$

is an $\left(\in, \in \vee \mathrm{q}_{k}\right)$-fuzzy normal subgroup of $\mathcal{G}(\mathcal{A})$.

Proof. Let $\mathcal{A}_{x}, \mathcal{A}_{y} \in \mathcal{G}(\mathcal{A})$. Using Theorems 4.5 and 5.5, we have

$$
\begin{aligned}
\tilde{\mathcal{A}}\left(\mathcal{A}_{x} \cdot \mathcal{A}_{y}\right) & =\tilde{\mathcal{A}}\left(\mathcal{A}_{x y}\right)=\mathcal{A}(x y) \\
& \geq \min \left\{\mathcal{A}(x), \mathcal{A}(y), \frac{1-k}{2}\right\} \\
& =\min \left\{\tilde{\mathcal{A}}\left(\mathcal{A}_{x}\right), \tilde{\mathcal{A}}\left(\mathcal{A}_{y}\right), \frac{1-k}{2}\right\}, \\
\tilde{\mathcal{A}}\left(\mathcal{A}_{x}^{-1}\right)=\tilde{\mathcal{A}}\left(\mathcal{A}_{x^{-1}}\right)=\mathcal{A}\left(x^{-1}\right) & \geq \min \left\{\mathcal{A}(x), \frac{1-k}{2}\right\}=\min \left\{\tilde{\mathcal{A}}\left(\mathcal{A}_{x}\right), \frac{1-k}{2}\right\},
\end{aligned}
$$

and

$$
\begin{aligned}
\tilde{\mathcal{A}}\left(\mathcal{A}_{x} \cdot \mathcal{A}_{y}\right) & =\tilde{\mathcal{A}}\left(\mathcal{A}_{x y}\right)=\mathcal{A}(x y) \\
& \geq \min \left\{\mathcal{A}(y x), \frac{1-k}{2}\right\} \\
& =\min \left\{\tilde{\mathcal{A}}\left(\mathcal{A}_{y x}\right), \frac{1-k}{2}\right\} \\
& =\min \left\{\tilde{\mathcal{A}}\left(\mathcal{A}_{y} \cdot \mathcal{A}_{x}\right), \frac{1-k}{2}\right\} .
\end{aligned}
$$

Hence $\tilde{\mathcal{A}}$ is an $\left(\in, \in \vee \mathrm{q}_{k}\right)$-fuzzy normal subgroup of $\mathcal{G}(\mathcal{A})$ by Theorems 4.5 and 5.5 . 
Theorem 5.14. For any $\left(\in, \in \vee q_{k}\right)$-fuzzy (normal) subgroup of $\mathcal{G}(\mathcal{A})$, there exists an $\left(\in, \in \vee \mathrm{q}_{k}\right)$-fuzzy (normal) subgroup of $G$.

Proof. Let $\mathcal{A}^{*}$ be an $\left(\in, \in \vee \mathrm{q}_{k}\right)$-fuzzy subgroup of $\mathcal{G}(\mathcal{A})$ and define a fuzzy subset $\mathcal{B}$ of $G$ by $\mathcal{B}(x)=\mathcal{A}^{*}\left(\mathcal{A}_{x}\right)$ for all $x \in G$. For any $x, y \in G$, we have

$$
\begin{aligned}
\mathcal{B}(x y) & =\mathcal{A}^{*}\left(\mathcal{A}_{x y}\right)=\mathcal{A}^{*}\left(\mathcal{A}_{x} \cdot \mathcal{A}_{y}\right) \\
\geq & \min \left\{\mathcal{A}^{*}\left(\mathcal{A}_{x}\right), \mathcal{A}^{*}\left(\mathcal{A}_{y}\right), \frac{1-k}{2}\right\} \\
& =\min \left\{\mathcal{B}(x), \mathcal{B}(y), \frac{1-k}{2}\right\}
\end{aligned}
$$

and

$$
\mathcal{B}\left(x^{-1}\right)=\mathcal{A}^{*}\left(\mathcal{A}_{x^{-1}}\right)=\mathcal{A}^{*}\left(\mathcal{A}_{x}^{-1}\right) \geq \min \left\{\mathcal{A}^{*}\left(\mathcal{A}_{x}\right), \frac{1-k}{2}\right\}=\min \left\{\mathcal{B}(x), \frac{1-k}{2}\right\} .
$$

Hence $\mathcal{B}$ is an $\left(\in, \in \vee \mathrm{q}_{k}\right)$-fuzzy subgroup of $G$. Next, assume that $\mathcal{A}^{*}$ is $(\in$, $\left.\in \vee \mathrm{q}_{k}\right)$-fuzzy normal. Then

$$
\begin{aligned}
\mathcal{B}(x y) & =\mathcal{A}^{*}\left(\mathcal{A}_{x y}\right)=\mathcal{A}^{*}\left(\mathcal{A}_{x} \cdot \mathcal{A}_{y}\right) \\
& \geq \min \left\{\mathcal{A}^{*}\left(\mathcal{A}_{y} \cdot \mathcal{A}_{x}\right), \frac{1-k}{2}\right\} \\
& =\min \left\{\mathcal{A}^{*}\left(\mathcal{A}_{y x}\right), \frac{1-k}{2}\right\} \\
& =\min \left\{\mathcal{B}(y x), \frac{1-k}{2}\right\} .
\end{aligned}
$$

It follows from Theorem 5.5 that $\mathcal{B}$ is $\left(\in, \in \vee \mathrm{q}_{k}\right)$-fuzzy normal.

Theorem 5.15. Let $\mathcal{A}$ be an $\left(\in, \in \vee \mathrm{q}_{k}\right)$-fuzzy subgroup of $G$ which is not an $(\in, \in)$-fuzzy subgroup of $G$. Let $H:=\left\{x \in G \mid \overleftarrow{\mathcal{A}}_{e} \subseteq \overleftarrow{\mathcal{A}}_{x}\right\}$. Then

(1) $H$ is a subgroup of $G$ and $H=\left\{x \in G \mid \overleftarrow{\mathcal{A}}_{e}=\overleftarrow{\mathcal{A}}_{x}\right\}$

(2) If $\mathcal{A}$ is $\left(\in, \in \vee \mathrm{q}_{k}\right)$-fuzzy normal, then $H$ is normal.

Proof. (1) If $x \in H$, then $\overleftarrow{\mathcal{A}}_{e} \subseteq \overleftarrow{\mathcal{A}}_{x}$ and so $\overleftarrow{\mathcal{A}}_{e}(y) \leq \overleftarrow{\mathcal{A}}_{x}(y)$ for all $y \in G$. It follows that

$$
\begin{aligned}
\mathcal{A}\left(y x^{-1}\right) & \geq \min \left\{\mathcal{A}\left(y x^{-1}\right), \frac{1-k}{2}\right\}=\min \left\{\mathcal{A}_{x}^{l}(y), \frac{1-k}{2}\right\} \\
& =\overleftarrow{\mathcal{A}}_{x}(y) \geq \overleftarrow{\mathcal{A}}_{e}(y)=\min \left\{\mathcal{A}_{e}^{l}(y), \frac{1-k}{2}\right\} \\
& =\min \left\{\mathcal{A}\left(y e^{-1}\right), \frac{1-k}{2}\right\}=\min \left\{\mathcal{A}(y), \frac{1-k}{2}\right\}
\end{aligned}
$$

for all $y \in G$. By taking $y=e$ and using Theorem 4.16, we have

$$
\mathcal{A}\left(x^{-1}\right) \geq \min \left\{\mathcal{A}(e), \frac{1-k}{2}\right\}=\frac{1-k}{2},
$$

and hence $\mathcal{A}(x) \geq \min \left\{\mathcal{A}\left(x^{-1}\right), \frac{1-k}{2}\right\}=\frac{1-k}{2}$, i.e., $x \in \mathcal{A}_{\frac{1-k}{2}}$. This shows that $H \subseteq \mathcal{A}_{\frac{1-k}{2}}$. Now let $x \in \mathcal{A}_{\frac{1-k}{2}}$. Then $\mathcal{A}(x) \geq \frac{1-k}{2}$, and so

$$
\begin{aligned}
\mathcal{A}\left(y x^{-1}\right) & \geq \min \left\{\mathcal{A}(y), \mathcal{A}\left(x^{-1}\right), \frac{1-k}{2}\right\} \\
& \geq \min \left\{\mathcal{A}(y), \min \left\{\mathcal{A}(x), \frac{1-k}{2}\right\}, \frac{1-k}{2}\right\} \\
& =\min \left\{\mathcal{A}(y), \frac{1-k}{2}\right\}
\end{aligned}
$$


for all $y \in G$. It follows that

$$
\overleftarrow{\mathcal{A}}_{x}(y)=\min \left\{\mathcal{A}\left(y x^{-1}\right), \frac{1-k}{2}\right\} \geq \min \left\{\mathcal{A}(y), \frac{1-k}{2}\right\}=\overleftarrow{\mathcal{A}}_{e}(y)
$$

for all $y \in G$ so that $\overleftarrow{\mathcal{A}}_{e} \subseteq \overleftarrow{\mathcal{A}}_{x}$, i.e., $x \in H$. Therefore $H=\mathcal{A}_{\underline{1-k}}$ which is a subgroup of $G$ by Theorem 4.10. Obviously, $\left\{x \in G \mid \overleftarrow{\mathcal{A}}_{e}=\overleftarrow{\mathcal{A}}_{x}\right\} \subseteq H$. Let $x \in H$. Then $\overleftarrow{\mathcal{A}}_{e}(e) \leq \overleftarrow{\mathcal{A}}_{x}(e)$, and so $\mathcal{A}(x) \geq \frac{1-k}{2}$. For any $y \in G$, we get

$$
\begin{aligned}
\overleftarrow{\mathcal{A}}_{e}(y) & =\min \left\{\mathcal{A}(y), \frac{1-k}{2}\right\}=\min \left\{\mathcal{A}\left(y x^{-1} x\right), \frac{1-k}{2}\right\} \\
& \geq \min \left\{\mathcal{A}\left(y x^{-1}\right), \mathcal{A}(x), \frac{1-k}{2}\right\} \\
& =\min \left\{\mathcal{A}\left(y x^{-1}\right), \frac{1-k}{2}\right\}=\overleftarrow{\mathcal{A}}_{x}(y)
\end{aligned}
$$

i.e., $\overleftarrow{\mathcal{A}}_{x} \subseteq \overleftarrow{\mathcal{A}}_{e}$. Hence $\overleftarrow{\mathcal{A}}_{x}=\overleftarrow{\mathcal{A}}_{e}$, and thus $H=\left\{x \in G \mid \overleftarrow{\mathcal{A}}_{e}=\overleftarrow{\mathcal{A}}_{x}\right\}$

(2) If $\mathcal{A}$ is $\left(\in, \in \vee \mathrm{q}_{k}\right)$-fuzzy normal, then $H=\mathcal{A}_{\frac{1-k}{2}}$ is normal by Theorem 5.7 .

Theorem 5.16. Let $\mathcal{A}$ be an $\left(\in, \in \vee \mathrm{q}_{k}\right)$-fuzzy subgroup of $G$ which is not an $(\in, \in)$-fuzzy subgroup of $G$. If $\mathcal{A}$ is $\left(\in, \in \vee \mathrm{q}_{k}\right)$-fuzzy normal, then the following assertion is valid.

$$
(\forall x, y \in G)\left(\overleftarrow{\mathcal{A}}_{x}=\overleftarrow{\mathcal{A}}_{y} \Rightarrow\left(\forall t \in\left(0, \frac{1-k}{2}\right]\right)\left(x \mathcal{A}_{t}=y \mathcal{A}_{t}\right)\right)
$$

Proof. Let $t \in\left(0, \frac{1-k}{2}\right]$ and $x, y \in G$ such that $\overleftarrow{\mathcal{A}}_{x}=\overleftarrow{\mathcal{A}}_{y}$. Then

$$
\begin{aligned}
\mathcal{A}\left(y x^{-1}\right) & \geq \min \left\{\mathcal{A}\left(y x^{-1}\right), \frac{1-k}{2}\right\}=\overleftarrow{\mathcal{A}}_{x}(y)=\overleftarrow{\mathcal{A}}_{y}(y) \\
& =\min \left\{\mathcal{A}\left(y y^{-1}\right), \frac{1-k}{2}\right\}=\min \left\{\mathcal{A}(e), \frac{1-k}{2}\right\} \\
& =\frac{1-k}{2} \geq t
\end{aligned}
$$

and so $y x^{-1} \in \mathcal{A}_{t}$. Hence $x \mathcal{A}_{t}=y \mathcal{A}_{t}$.

The converse of Theorem 5.16 is not true as shown by the next example.

Example 5.17. Consider the dihedral group

$$
D_{4}=\left\{1, \sigma, \sigma^{2}, \sigma^{3}, \tau, \sigma \tau, \sigma^{2} \tau, \sigma^{3} \tau\right\}
$$

of order 8 where the multiplication is defined by Table 4 . Let $\mathcal{A}$ be a fuzzy subset of $G$ defined by

$$
\mathcal{A}=\left(\begin{array}{cccccccc}
1 & \sigma & \sigma^{2} & \sigma^{3} & \tau & \sigma \tau & \sigma^{2} \tau & \sigma^{3} \tau \\
0.4 & 0.3 & 0.7 & 0.3 & 0.2 & 0.2 & 0.2 & 0.2
\end{array}\right)
$$

Then $\mathcal{A}$ is an $\left(\in, \in \vee \mathrm{q}_{k}\right)$-fuzzy normal subgroup of $G$ for $k=0.26$, and $\mathcal{A}_{0.3}=$ $\left\{1, \sigma, \sigma^{2}, \sigma^{3}\right\}$. Hence $\tau \mathcal{A}_{0.3}=\left\{\tau, \sigma \tau, \sigma^{2} \tau, \sigma^{3} \tau\right\}=\sigma^{3} \tau \mathcal{A}_{0.3}$. But $\overleftarrow{\mathcal{A}}_{\tau}(\tau)=0.37$ and $\overleftarrow{\mathcal{A}}_{\sigma^{3} \tau}(\tau)=0.3$, and so $\overleftarrow{\mathcal{A}}_{\tau} \neq \overleftarrow{\mathcal{A}}_{\sigma^{3} \tau}$ 
TABLE 4. Multiplication table for $D_{4}$

\begin{tabular}{c|llllllll}
\hline & 1 & $\sigma$ & $\sigma^{2}$ & $\sigma^{3}$ & $\tau$ & $\sigma \tau$ & $\sigma^{2} \tau$ & $\sigma^{3} \tau$ \\
\hline 1 & 1 & $\sigma$ & $\sigma^{2}$ & $\sigma^{3}$ & $\tau$ & $\sigma \tau$ & $\sigma^{2} \tau$ & $\sigma^{3} \tau$ \\
$\sigma$ & $\sigma$ & $\sigma^{2}$ & $\sigma^{3}$ & 1 & $\sigma \tau$ & $\sigma^{2} \tau$ & $\sigma^{3} \tau$ & $\tau$ \\
$\sigma^{2}$ & $\sigma^{2}$ & $\sigma^{3}$ & 1 & $\sigma$ & $\sigma^{2} \tau$ & $\sigma^{3} \tau$ & $\tau$ & $\sigma \tau$ \\
$\sigma^{3}$ & $\sigma^{3}$ & 1 & $\sigma$ & $\sigma^{2}$ & $\sigma^{3} \tau$ & $\tau$ & $\sigma \tau$ & $\sigma^{2} \tau$ \\
$\tau$ & $\tau$ & $\sigma^{3} \tau$ & $\sigma^{2} \tau$ & $\sigma \tau$ & 1 & $\sigma^{3}$ & $\sigma^{2}$ & $\sigma$ \\
$\sigma \tau$ & $\sigma \tau$ & $\tau$ & $\sigma^{3} \tau$ & $\sigma^{2} \tau$ & $\sigma$ & 1 & $\sigma^{3}$ & $\sigma^{2}$ \\
$\sigma^{2} \tau$ & $\sigma^{2} \tau$ & $\sigma \tau$ & $\tau$ & $\sigma^{3} \tau$ & $\sigma^{2}$ & $\sigma$ & 1 & $\sigma^{3}$ \\
$\sigma^{3} \tau$ & $\sigma^{3} \tau$ & $\sigma^{2} \tau$ & $\sigma \tau$ & $\tau$ & $\sigma^{3}$ & $\sigma^{2}$ & $\sigma$ & 1 \\
\hline
\end{tabular}

Theorem 5.18. Let $\mathcal{A}$ be a fuzzy subset of $G$ and

$$
H:=\left\{x \in G \mid \mathcal{A}(x) \geq \frac{1-k}{2}\right\} .
$$

If $\mathcal{A}$ is an $\left(\in, \in \vee \mathrm{q}_{k}\right)$-fuzzy subgroup of $G$ which is not an $(\in, \in)$-fuzzy subgroup of $G$, then

$$
(\forall x, y \in G)\left(H x=H y \Rightarrow \overleftarrow{\mathcal{A}}_{x}=\overleftarrow{\mathcal{A}}_{y}\right)
$$

Proof. Note that $H=\mathcal{A}_{\underline{\underline{1-k}}}$ which is a subgroup of $G$. It follows from Theorem 5.15 that $H=\left\{x \in G \mid \frac{\stackrel{1}{\mathcal{A}}_{e}}{2}=\overleftarrow{\mathcal{A}}_{x}\right\}$. Assume that $H x=H y$. Then $x y^{-1} \in H$ and so $\overleftarrow{\mathcal{A}}_{e}=\overleftarrow{\mathcal{A}}_{x y^{-1}}$, i.e.

$$
\min \left\{\mathcal{A}(z), \frac{1-k}{2}\right\}=\min \left\{\mathcal{A}\left(z y x^{-1}\right), \frac{1-k}{2}\right\}
$$

for all $z \in G$. If we take $z=z y^{-1}$ in (5.5), then

$$
\overleftarrow{\mathcal{A}}_{y}(z)=\min \left\{\mathcal{A}\left(z y^{-1}\right), \frac{1-k}{2}\right\}=\min \left\{\mathcal{A}\left(z x^{-1}\right), \frac{1-k}{2}\right\}=\overleftarrow{\mathcal{A}}_{x}(z)
$$

for all $z \in G$. Therefore $\overleftarrow{\mathcal{A}}_{x}=\overleftarrow{\mathcal{A}}_{y}$

\section{References}

[1] S. K. Bhakat, $(\in \vee q)$-level subset, Fuzzy Sets and Systems 103 (1999), no. 3, 529-533.

$[2] \_,(\in, \in \vee q)$-fuzzy normal, quasinormal and maximal subgroups, Fuzzy Sets and Systems 112 (2000), no. 2, 299-312.

[3] S. K. Bhakat and P. Das, On the definition of a fuzzy subgroup, Fuzzy Sets and Systems 51 (1992), no. 2, 235-241

[4] —,$(\in, \in \vee q)$-fuzzy subgroup, Fuzzy Sets and Systems 80 (1996), no. 3, 359-368.

[5] V. N. Dixit, R. Kumar, and N. Ajmal, Level subgroups and union of fuzzy subgroups, Fuzzy Sets and Systems 37 (1990), no. 3, 359-371.

[6] D. Dubois and H. Prade, Gradual elements in a fuzzy set, Soft Comput. 12 (2008), $165-175$.

[7] T. Head, A metatheorem for deriving fuzzy theorems from crisp versions, Fuzzy Sets and Systems 73 (1995), no. 3, 349-358.

[8] W.-J. Liu, Fuzzy invariant subgroups and fuzzy ideals, Fuzzy Sets and Systems 8 (1982), no. $2,133-139$. 
[9] P. K. Maji, A. R. Roy, and R. Biswas, An application of soft sets in a decision making problem, Comput. Math. Appl. 44 (2002), no. 8-9, 1077-1083.

[10] D. Molodtsov, Soft set theory-first results, Comput. Math. Appl. 37 (1999), no. 4-5, 19-31.

[11] N. P. Mukherjee and P. Bhattacharya, Fuzzy normal subgroups and fuzzy cosets, Inform. Sci. 34 (1984), no. 3, 225-239.

[12] __ Fuzzy groups: some group-theoretic analogs, Inform. Sci. 39 (1986), no. 3, 247268.

[13] V. Murali, Fuzzy points of equivalent fuzzy subsets, Inform. Sci. 158 (2004), 277-288.

[14] P. M. Pu and Y. M. Liu, Fuzzy topology. I. Neighborhood structure of a fuzzy point and Moore-Smith convergence, J. Math. Anal. Appl. 76 (1980), no. 2, 571-599.

[15] A. Rosenfeld, Fuzzy groups, J. Math. Anal. Appl. 35 (1971), 512-517.

[16] X. Yuan, C. Zhang, and Y. Ren, Generalized fuzzy groups and many-valued implications, Fuzzy Sets and Systems 138 (2003), no. 1, 205-211.

[17] L. A. Zadeh, Fuzzy sets, Information and Control 8 (1965), 338-353.

Young Bae Jun

Department of Mathematics Education (and Rins)

Gyeongsang National University

Chinju 660-701, Korea

E-mail address: skywine@gmail.com

Min Su Kang

Department of Mathematics

HANYANG UNIVERSITY

SeOul 133-791, Korea

E-mail address: sinchangmyun@hanmail.net

Chul Hwan Park

School of Digital, Mechanics

Ulsan College

ULSAN 680-749,Korea

E-mail address: skyrosemary@gmail.com 